\title{
QUEEN'S
QNEIVERSITY
BELFAST
}

\section{Physical-Layer Security for Frequency Diverse Array-Based Directional Modulation in Fluctuating Two-Ray Fading Channels}

Cheng, Q., Wang, S., Fusco, V., Wang, F., Zhu, J., \& Gu, C. (2021). Physical-Layer Security for Frequency Diverse Array-Based Directional Modulation in Fluctuating Two-Ray Fading Channels. IEEE Transactions on Wireless Communications.

Published in:

IEEE Transactions on Wireless Communications

Document Version:

Peer reviewed version

Queen's University Belfast - Research Portal:

Link to publication record in Queen's University Belfast Research Portal

Publisher rights

(c) 2021 IEEE.

This work is made available online in accordance with the publisher's policies. Please refer to any applicable terms of use of the publisher.

\section{General rights}

Copyright for the publications made accessible via the Queen's University Belfast Research Portal is retained by the author(s) and / or other copyright owners and it is a condition of accessing these publications that users recognise and abide by the legal requirements associated with these rights.

Take down policy

The Research Portal is Queen's institutional repository that provides access to Queen's research output. Every effort has been made to ensure that content in the Research Portal does not infringe any person's rights, or applicable UK laws. If you discover content in the Research Portal that you believe breaches copyright or violates any law, please contact openaccess@qub.ac.uk. 


\title{
Physical-Layer Security for Frequency Diverse Array-Based Directional Modulation in Fluctuating Two-Ray Fading Channels
}

\author{
Qian Cheng, Shilian Wang, Member, IEEE, Vincent Fusco, Fellow, IEEE, \\ Fanggang Wang, Senior Member, IEEE, Jiang Zhu, Member, IEEE, and Chao Gu
}

\begin{abstract}
The frequency diverse array (FDA)-based directional modulation (DM) technology plays an important role in the physical-layer security (PLS) transmission of 5G and beyond communications. In order to meet the tremendous increase in mobile data traffic, a new memory-efficient design for the FDADM-based PLS transmission is urgently demanded. In this paper, an analytical symmetrical multi-carrier FDA model is proposed in three dimensions, namely, range, azimuth angle, and elevation angle, differing from the conventional analytical approach with only range and azimuth angle considered. Then, a single-point (SP) artificial noise (AN) aided FDA-DM scheme is proposed, which reduces the memory consumption significantly compared with the conventional zero-forcing $(Z F)$ and singular value decomposition (SVD) approaches. Moreover, the PLS performance of the proposed FDA-DM scheme is analyzed in fluctuating tworay (FTR) fading channels for the first time, including the average secrecy capacity (ASC) and the secrecy outage probability (SOP). More importantly, the closed-form expressions for the lower bound on ASC and the upper bound on SOP are derived, respectively. The effectiveness of the analytical expressions is verified by numerical simulations. This work opens a way to lower the memory requirements for DM-based PLS transmission of $5 \mathrm{G}$ and beyond communications.
\end{abstract}

Index Terms-Directional modulation; fluctuating two-ray fading; frequency diverse array; physical-layer security; secrecy capacity; secrecy outage probability.

\section{INTRODUCTION}

$\mathbf{P}$ HYSICAL-LAYER security (PLS) technology, as a promising and powerful alternative to complement and even possibly replace encryption-based approaches, has been brought to the forefront by plenty of researchers [1]-[3]. The

This work was supported in part by the National Key R\&D Program of China under Grant 2020YFB1806903, inpart by China Scholarship Council (CSC) under Grant 201803170247, and in part by the National Natural Science Foundation under Grant U1834210. The associate editor coordinating the review of this article and approving it for publication was Nan Yang. (Corresponding Author: Shilian Wang; Vincent Fusco.)

Q. Cheng was with the College of Electronic Science and Technology, National University of Defense Technology, Changsha 410073, China; He is now with Systems Engineering Institute, Army Research Academy, Beijing 100072, China. (e-mail: Q.Cheng27@ foxmail.com).

S. Wang and J. Zhu are with the College of Electronic Science and Technology, National University of Defense Technology, Changsha 410073, China (e-mail: \{wangsl, jiangzhu\}@ @udt.edu.cn).

V. Fusco and C. Gu are with the Institute of Electronics, Communications and Information Technology (ECIT), Queen's University Belfast, Belfast BT3 9DT, U.K. (e-mail: v.fusco@ecit.qub.ac.uk, chao.gu@qub.ac.uk).

F. Wang is with the State Key Laboratory of Rail Traffic Control and Safety, Beijing Jiaotong University, Beijing 100044, China (e-mail: wangfg@bjtu.edu.cn).

Digital Object Identifier 10.1109/TWC.2021.3056521 key idea of PLS is to fully exploit the characteristics of wireless channels to secure the transmission link from the legitimate transmitter to the desired receiver in the presence of unauthorized eavesdroppers [4], [5]. Owing to the promising benefits of PLS technology, it has been intensively investigated to safeguard $5 \mathrm{G}$ and beyond wireless communications [6], [7]. However, implementing the PLS transmission can result in considerable memory consumptions, which imposes stringent requirements on the $5 \mathrm{G}$ nodes or devices. In addition, the tremendously increasing mobile data traffic also requires considerable memory consumptions. Therefore, it is highly necessary to lower the memory consumption of the PLS transmission strategy for 5G and beyond communication system.

The directional modulation (DM) technology [8], which is capable of steering the standard baseband symbols along a desired direction while simultaneously distorting the received signals along other directions, has been regarded as a useful PLS transmission strategy for $5 \mathrm{G}$ millimeter-wave (mmWave) wireless communications [9], [10]. Traditionally, DM technology is implemented using phased arrays (PA) [11], which only achieves one-dimension security in the direction while loses security if the eavesdropper is in the same direction as the legitimate receiver. Compared with the PA-based DM technology, the frequency diverse array (FDA), exhibiting an extra range-dimension dependence apart from angle [12], [13], has been applied into DM implementations to realize twodimension security in both range and angle.

Specifically, the FDA was first utilized in [14] to achieve range-angle dependent secure DM transmissions with fixed linear frequency increments. The work in [15] utilized the FDA with non-linear frequency increments to decouple rangeand angle-dependent transmit beam patterns for DM transmissions. The FDAs with random and time-modulated frequency increments were exploited for secure DM transmissions in [16] and [17], respectively. FDA was also used in [18], [19] to establish secure DM transmissions for proximal legitimate user and eavesdropper. In addition to the single-user FDADM schemes [14]-[19], multi-user FDA-DM schemes were also investigated intensively by means of the spread spectrum technology [20], optimization algorithms [21], singular value decomposition (SVD) [22], weighted-type fractional Fourier transform (WFRFT) [23], and cooperative-receiver index modulation [24], respectively. Inspired by the frequency diversity, the works in [25], [26] designed a random-subcarrier- 
selection method for DM synthesis, which achieved rangeangle dependent secure precise transmission as well.

In the DM transmission schemes, artificial noise (AN) plays an important role [4]. Most of the AN-aided DM transmission schemes employ zero-forcing (ZF) method to design the orthogonal precoding matrix to remove the interference of AN for legitimate receivers [16], [18], [21], [27]-[29]. The SVD method provided another way to redesign the orthogonal precoding matrix [22]. These ZF or SVD-aided design approaches, however, consume too much memory to store the designed orthogonal matrix and AN. It still remains a challenge to design a memory-efficient secure DM transmission scheme for $5 \mathrm{G}$ and beyond communication systems. Moreover, the afore-mentioned DM-related works [14]-[29] only considered the line-of-sight $(\mathrm{LoS})$ channels in free space. Regarding the FDA-DM transmission in multipath fading channels, the authors in [30], [31] and [32] investigated the PLS performance of the FDA-DM communication system in Rayleigh and Nakagami- $m$ fading channels, respectively.

However, on the one hand, the works in [30]-[32] about FDA-DM transmissions in fading channels utilized ZF-based AN method, which demands high memory requirements as well. On the other hand, these conventional fading models like Rayleigh, Rician and Nakagami- $m$ fading cannot accurately fit the random small-scale fluctuations in real communication environments [33]. Recently, the fluctuating two-ray (FTR) fading model was proposed in [33], [34], which is a generalization of the existing fading models and can provide a better fit for small-scale fading measurements in mmWave communications. The authors in [35], [36] and [37] generalized the FTR fading model into arbitrary fading parameter case, cascaded case, and squared case, respectively. More recently, the average secrecy capacity (ASC), the secrecy outage probability (SOP) and the symbol error rate (SER) of FTR fading channels were analyzed in [38], [39] and [40] without AN (NoAN), respectively. The power adaption algorithm and wireless-powered UAV relay communication in FTR fading channels were investigated in [41] and [42], respectively.

To the best of our knowledge, there is no specific work in the state-of-the-art that aims to reduce the memory consumption of the FDA-DM scheme for $5 \mathrm{G}$ and beyond communications and to analyze the PLS performance of the FDA-DM scheme in FTR fading channels. We are the first to make this effort by proposing a memory-efficient single-point (SP) AN-aided FDA-DM scheme for $5 \mathrm{G}$ and beyond communications, and analyzing its PLS performance in FTR fading channels for the fist time. Overall, the main contributions of our work are as follows:

1) Different from the conventional analytical approach which only considers range and azimuth angle dimensions, an analytical model for the symmetrical multicarrier FDA is proposed in three dimensions, i.e., range, azimuth angle and elevation angle.

2) A memory-efficient FDA-DM scheme is further proposed with the assistance of single-point AN, which significantly outperforms the conventional ZF method [16], [18], [21], [27]-[32] and the SVD method [22]. The

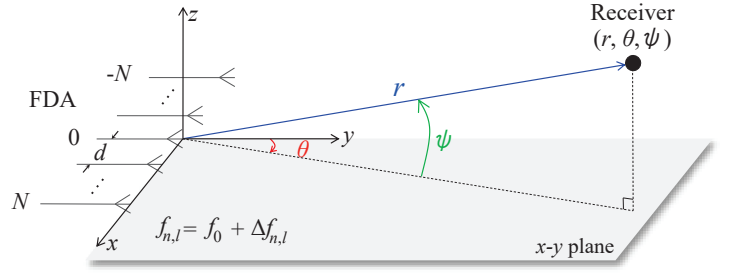

Fig. 1. The proposed FDA-DM scheme in FTR fading channels.

proposed memory-efficient FDA-DM scheme provides an efficient strategy to lower the memory requirements for the PLS transmissions of 5G and beyond communications.

3) The ASC and the SOP of the proposed FDA-DM scheme are analyzed in FTR fading channels for the first time. We also derive the closed-form expressions for the lower bound on ASC and the upper bound on SOP. Numerical experiments are conducted to compare the PLS performances of the proposed $\mathrm{SP}$ and the conventional $\mathrm{ZF}$ [16], [18], [21], [27]-[32], SVD [22] and NoAN [38][40] methods.

The remainder of this paper is organized as follows. Section II proposes an analytical model of symmetrical multicarrier FDA in three dimensions. A memory-efficient FDADM scheme is proposed in Section III with the assistance of single-point AN, where the comparison between the proposed SP method and the conventional ZF and SVD methods is also provided. Section IV analyzes the ASC and the SOP performances of the proposed FDA-DM scheme in FTR fading channels. Numerical results are conducted in Section V in order to verify the advantages of the proposed FDA-DM scheme. Finally, Section VI makes a conclusion for the whole paper and points out the future work.

Notations: In this paper, $\imath=\sqrt{-1}$ indicates imaginary unit. The operators $(\cdot)^{\mathrm{T}}$ and $(\cdot)^{\mathrm{H}}$ represent the transpose and the Hermitian transpose of a matrix. The set of complex numbers is denoted by $\mathbb{C}$. The notation $\mathbb{E}(\cdot)$ refers to the expectation of a random variable, while $\operatorname{tr}(\cdot)$ represents the trace of a matrix. In addition, $\max \{\cdot\}$ and $|\cdot|$ refer to the maximum value of a set of real numbers and the modulus of a complex number, respectively. $\mathcal{N}\left(0, \sigma^{2}\right)$ and $\mathcal{C N}\left(0, \sigma^{2}\right)$ refer to the real and complex Gaussian distributions with zero mean and variance $\sigma^{2}$, respectively. $\mathcal{U}[\cdot, \cdot)$ is the uniform distribution. Finally, the probability function is denoted by $\operatorname{Pr}(\cdot)$.

\section{FDA Model IN Three Dimensions}

Most of the state-of-the-art analyzes the FDA only in two dimensions [12]-[23], namely, range and azimuth angle. In this paper, we take an extra dimension, elevation angle, into consideration and establish an analytical FDA model in three dimensions, i.e., range, azimuth angle, and elevation angle. Different from the conventional rectangular/circular antenna arrays that use the "space diversity" (planar antennas) to generate 3-dimension coordinate [43], the linear frequency diverse arrays utilize the "frequency diversity" (different frequencies on different antennas) to produce range-angle dependent 3dimension beampatterns [44]. 
As shown in Fig. 1, the FDA consists of $2 N+1$ antenna elements with equal element spacing $d$, which is set as half wavelength of the central carrier. These elements are symmetrically and linearly arrayed on the $x$-axis with the central element located at the coordinate origin. For each element, there are $L$ subcarriers to transmit. The radiated frequency of the $l$-th $(l=0, \cdots, L-1)$ subcarrier of the $n$-th $(n=-N, \cdots, 0, \cdots, N)$ element is designed as

$$
\begin{aligned}
f_{n, l} & =f_{0}+\Delta f_{n, l} \\
& =f_{0}+\Delta f \ln (|n|+1) \ln (l+1),
\end{aligned}
$$

where $f_{0}$ is the central radiated frequency, $\Delta f$ refers to a fixed frequency increment, and $\Delta f_{n, l}=\Delta f \ln (|n|+1) \ln (l+1)$ represents the frequency increment between the central frequency and the $l$-th subcarrier of the $n$-th element, which satisfies

$$
\left|\Delta f_{n, l}\right| \ll f_{0} \text {. }
$$

In order to derive the steering vector of the FDA, we consider that each element of the transmitter transmits sinusoidal signals with $L$ subcarriers. The $l$-th subcarrier signal transmitted by the $n$-th element at time $t$ can be expressed as

$$
x_{n, l}(t)=\exp \left\{\imath 2 \pi f_{n, l} t\right\} .
$$

Let $r, \theta$ and $\psi$ represent the range, azimuth angle and elevation angle, respectively, as shown in Fig. 1. For an arbitrary receiver located at $(r, \theta, \psi)$, the overall observed signal in the far field can be written as

$$
\begin{aligned}
y(r, \theta, \psi) & =\sum_{n=-N}^{N} \sum_{l=0}^{L-1} x_{n, l}\left(t-\frac{r_{n}}{c}\right) \\
& =\sum_{n=-N}^{N} \sum_{l=0}^{L-1} \exp \left\{\imath 2 \pi f_{n, l}\left(t-\frac{r_{n}}{c}\right)\right\},
\end{aligned}
$$

where $c$ denotes light speed and $r_{n}$ refers to the path length from the $n$-th element to the observation point. With some basic geometric operations, the path length $r_{n}$ can be approximated by [44]

$$
r_{n} \approx r-n d \sin \theta \cos \psi
$$

Taking (1) and (5) into (4b) yields (6). The constraint in (2) implies that the last term in the summation, $\Delta f_{n, l} n d \sin \theta \cos \psi / c$, can be omitted, so (6) can be further approximated as

$$
\begin{aligned}
& y(r, \theta, \psi) \approx \exp \left\{\imath 2 \pi f_{0}\left(t-\frac{r}{c}\right)\right\} \\
& \times \sum_{n=-N}^{N} \sum_{l=0}^{L-1} \exp \left\{\imath 2 \pi\left[\Delta f_{n, l}\left(t-\frac{r}{c}\right)+\frac{f_{0} n d \sin \theta \cos \psi}{c}\right]\right\} .
\end{aligned}
$$

The terms inside the summation of (7) are decided by the geometry and the frequency-offset scheme of the FDA. Therefore, the sub-steering vector caused by the $L$ subcarriers of the $n$-th antenna element can be written as [45]

$\mathbf{a}_{n}(r, \theta, \psi)=\left[\exp \left\{\phi_{n, 0}\right\} \cdots \exp \left\{\phi_{n, l}\right\} \cdots \exp \left\{\phi_{n, L-1}\right\}\right]^{\mathrm{T}}$,

which is a vector with size $L \times 1$. The $l$-th phase term $\phi_{n, l}$ in (8) is given by

$$
\phi_{n, l}=\imath 2 \pi\left[\Delta f_{n, l}\left(t-\frac{r}{c}\right)+\frac{f_{0} n d \sin \theta \cos \psi}{c}\right] .
$$

Overall, the normalized steering vector of the symmetrical multi-carrier FDA can be obtained by combining the $2 N+1$ sub-steering vectors $\mathbf{a}_{n}(r, \theta, \psi)$, i.e.,

$$
\begin{aligned}
\mathbf{h}(r, \theta, \psi)= & \frac{1}{\sqrt{(2 N+1) L}} \\
& \times\left[\mathbf{a}_{-N}^{\mathrm{T}}(r, \theta, \psi) \cdots \mathbf{a}_{n}^{\mathrm{T}}(r, \theta, \psi) \cdots \mathbf{a}_{N}^{\mathrm{T}}(r, \theta, \psi)\right]_{10)}^{\mathrm{T}},
\end{aligned}
$$

which is a vector with size $(2 N+1) L \times 1$, and satisfies $\mathbf{h}^{\mathrm{H}}(r, \theta, \psi) \mathbf{h}(r, \theta, \psi)=1$.

\section{LOW-MEMORY-CONSUMPTION FDA-DM SCHEME IN FTR FADING CHANNELS}

We consider a multi-input single-output single-eavesdropper (MISOSE) wiretap channel model, where both the legitimate receiver (Bob) and the eavesdropper (Eve) are equipped with a single antenna. A legitimate transmitter (Alice), equipped with a $(2 N+1)$-element symmetrical FDA with each element having $L$ subcarriers, intends to deliver confidential information to Bob, while a malicious Eve at a different location tries to crack the confidential information via its received signal. The coordinate origin is set as the central element of Alice's FDA, as analyzed in Section II. We also assume both Bob's and Eve's channels undergo independent and quasi-static FTR fading.

\section{A. Alice's Transmit Signal}

The transmit signal of Alice consists of two parts. The first is the normalized baseband modulation symbol $s \in \Omega$ and $\mathbb{E}\left(|s|^{2}\right)=1$, where $\Omega$ is the alphabet of the baseband modulation symbols with the size of $M$. The second is the inserted single-point $\mathrm{AN} z$, which follows a complex Gaussian distribution, i.e., $z \sim \mathcal{C N}(0,1)$. To match the $(2 N+1) L$ FDA subcarriers of Alice, the baseband symbol $s$ and the inserted AN $z$ should be precoded with a normalization vector $\mathbf{p}_{1}$ and an orthogonal vector $\mathbf{p}_{2}$, respectively, which require

$$
\mathbf{h}_{\mathrm{B}}^{\mathrm{H}} \mathbf{p}_{1}=1
$$

$$
\begin{aligned}
y(r, \theta, \psi) & \approx \sum_{n=-N}^{N} \sum_{l=0}^{L-1} \exp \left\{\imath 2 \pi\left(f_{0}+\Delta f_{n, l}\right)\left(t-\frac{r-n d \sin \theta \cos \psi}{c}\right)\right\} \\
& =\exp \left\{\imath 2 \pi f_{0}\left(t-\frac{r}{c}\right)\right\} \sum_{n=-N}^{N} \sum_{l=0}^{L-1} \exp \left\{\imath 2 \pi\left[\Delta f_{n, l}\left(t-\frac{r}{c}\right)+\frac{f_{0} n d \sin \theta \cos \psi}{c}+\frac{\Delta f_{n, l} n d \sin \theta \cos \psi}{c}\right]\right\} .
\end{aligned}
$$


TABLE I

COMPARISON FOR MEMORY REQUIREMENTS OF DIFFERENT FDA-DM SCHEMES

\begin{tabular}{cccc}
\hline Items & $\mathbf{Z F}[16],[18],[21],[27]-[32]$ & SVD [22] & Proposed SP \\
\hline Orthogonal matrix/vector & $\mathbf{P}_{2}^{\mathrm{ZF}}=\mathbf{I}_{(2 N+1) L}-\mathbf{h}_{\mathrm{B}} \mathbf{h}_{\mathrm{B}}^{\mathrm{H}}$ & $\mathbf{h}_{\mathrm{B}}^{\mathrm{H}}=\mathbf{U}[\mathbf{D} \mathbf{0}]\left[\mathbf{V}_{1} \mathbf{V}_{0}\right]^{\mathrm{H}}, \mathbf{P}_{2}^{\mathrm{SVD}}=\mathbf{V}_{0}$ & $\mathbf{p}_{2}^{\mathrm{SP}}=$ Orthogonal vector of $\mathbf{h}_{\mathrm{B}}^{\mathrm{H}}$ \\
Size of orthogonal matrix/vector & $(2 N+1) L \times(2 N+1) L$ & $(2 N+1) L \times 2 N$ & $(2 N+1) L \times 1$ \\
Artificial noise & $\mathbf{z}^{\mathrm{ZF}} \in \mathbb{C}^{(2 N+1) L \times 1}$ & $\mathbf{z}^{\mathrm{SVD}} \in \mathbb{C}^{2 N \times 1}$ & $z^{\mathrm{SP}} \in \mathbb{C}$ \\
Size of artificial noise & $(2 N+1) L \times 1$ & $2 N \times 1$ & 1 \\
Total size & $(2 N+1)^{2} L^{2}+(2 N+1) L$ & $2 N(2 N+1) L+2 N$ & $(2 N+1) L+1$ \\
Memory complexity & $\mathcal{O}\left(N^{2} L^{2}\right)$ & $\mathcal{O}\left(N^{2} L\right)$ & $\mathcal{O}(N L)$ \\
\hline
\end{tabular}

and

$$
\mathbf{h}_{\mathrm{B}}^{\mathrm{H}} \mathbf{p}_{2}=0,
$$

where $\mathbf{h}_{\mathrm{B}}=\mathbf{h}\left(r_{\mathrm{B}}, \theta_{\mathrm{B}}, \psi_{\mathrm{B}}\right)$ is the normalized steering vector at Bob's location, $\left(r_{\mathrm{B}}, \theta_{\mathrm{B}}, \psi_{\mathrm{B}}\right)$. Since the angle and range estimation algorithms, such as [48], [49], could be applied to help Alice estimate Bob's location, we assume $\left(r_{\mathrm{B}}, \theta_{\mathrm{B}}, \psi_{\mathrm{B}}\right)$ is a priori perfectly known at Alice. If the practical measurement errors are considered, the robust method in [27] could be useful to improve the robustness of the proposed DM scheme.

Therefore, the transmitting signal vector which feeds Alice's $2 N+1$ antenna elements can be expressed as

$$
\mathbf{x}=\beta_{1} \sqrt{P_{s}} \mathbf{p}_{1} s+\alpha \beta_{2} \sqrt{P_{s}} \mathbf{p}_{2} z,
$$

where $P_{s}$ is the total transmitting power; $\alpha=1 / \sqrt{\operatorname{tr}\left(\mathbf{p}_{2} \mathbf{p}_{2}^{\mathrm{H}}\right)}$ is the power normalization factor for the inserted $\mathrm{AN} ; \beta_{1}$ and $\beta_{2}$ are power splitting factors for the baseband symbol and the inserted $\mathrm{AN}$, respectively, which satisfy the following constraint,

$$
\beta_{1}^{2}+\beta_{2}^{2}=1
$$

For the design of $\mathbf{p}_{1}$ and $\mathbf{p}_{2}$, we first review the $\mathrm{ZF}$ method [16], [18], [21], [27]-[32] and the SVD method [22] briefly, and then propose a memory-efficient SP method.

1) ZF method: The normalization vector is directly designed as the normalized steering vector at Bob's location, i.e., $\mathbf{p}_{1}^{\mathrm{ZF}}=$ $\mathbf{h}_{\mathrm{B}}$. In addition, the orthogonal matrix of the $\mathrm{ZF}$ method is designed as $\mathbf{P}_{2}^{\mathrm{ZF}}=\mathbf{I}_{(2 N+1) L}-\mathbf{h}_{\mathrm{B}} \mathbf{h}_{\mathrm{B}}^{\mathrm{H}}$, which is actually a matrix with the size of $(2 N+1) L \times(2 N+1) L$. In order to match the orthogonal matrix $\mathbf{P}_{2}^{\mathrm{ZF}}$, the inserted $\mathrm{AN}$ of the $\mathrm{ZF}$ method should be randomly valued from $\mathbf{z}^{\mathrm{ZF}} \in \mathbb{C}^{(2 N+1) L \times 1}$.

2) SVD method: Similar to the ZF method, the normalization vector of the SVD method is also designed as $\mathbf{p}_{1}^{\mathrm{SVD}}=\mathbf{h}_{\mathrm{B}}$. But for the design of the orthogonal matrix, the SVD method first solves the SVD of $\mathbf{h}_{\mathrm{B}}^{\mathrm{H}}$, i.e., $\mathbf{h}_{\mathrm{B}}^{\mathrm{H}}=\mathbf{U}\left[\begin{array}{ll}\mathbf{D} & \mathbf{0}\end{array}\right]\left[\begin{array}{ll}\mathbf{V}_{1} & \mathbf{V}_{0}\end{array}\right]^{\mathrm{H}}$, from which a null space of $\mathbf{h}_{\mathrm{B}}^{\mathrm{H}}$ can be obtained. Then, the orthogonal matrix $\mathbf{P}_{2}^{\mathrm{SVD}}$ can be directly designed as $\mathbf{P}_{2}^{\mathrm{SVD}}=\mathbf{V}_{0}$, which is a matrix with the size of $(2 N+1) L \times 2 N$. Consequently, the inserted AN of the SVD method has to be changed as $\mathbf{z}^{\mathrm{SVD}} \in \mathbb{C}^{2 N \times 1}$ in order to match the orthogonal matrix $\mathbf{P}_{2}^{\mathrm{SVD}}$.

3) Proposed SP method: The normalization vector in this paper is designed as $\mathbf{p}_{1}^{\mathrm{SP}}=\mathbf{h}_{\mathrm{B}}$ as well. Different from the ZF and SVD methods which insert an AN vector in the transmit signal, the proposed SP method only requires a singlepoint AN as shown in (13). Therefore, the orthogonal vector $\mathbf{p}_{2}^{\mathrm{SP}}$ can be designed as an arbitrary orthogonal vector of $\mathbf{h}_{\mathrm{B}}^{\mathrm{H}}$ rather than a matrix. Comparatively, $\mathbf{p}_{2}^{\mathrm{SP}}$ can be an arbitrary column vector of $\mathbf{P}_{2}^{Z F}$ or $\mathbf{P}_{2}^{\mathrm{SVD}}$. In the following analysis, $\mathbf{p}_{2}$ specifically refers to the proposed $\mathbf{p}_{2}^{\mathrm{SP}}$.

4) Comparison for the $Z F$, SVD and SP methods: In order to illustrate the advantage of the the proposed SP method, Table I compares these three different design methods in terms of memory consumption to store the orthogonal matrix/vector and the AN. From Table I, the proposed SP method reduces the memory complexity from $\mathcal{O}\left(N^{2} L^{2}\right)$ and $\mathcal{O}\left(N^{2} L\right)$ to $\mathcal{O}(N L)$, which significantly outperforms the ZF and SVD methods.

In addition, Figs. 2(a) and (b) depict the numerical results for the total memory required and the ratio of SP method to $\mathrm{ZF}$ or SVD methods versus $N$ and $L$, respectively, which verify the excellent advantage of low memory consumption for the proposed SP method. For example, when $N=10$ and $L=7$, the proposed SP method only requires approximately $0.6803 \%$ total memory of the ZF method or $5 \%$ total memory of the SVD method. Figs. 3(a) and (b) further show the total memory consumptions and the corresponding ratios versus $N$ and $L$, respectively, which demonstrates that the proposed SP method can save much more memory than the conventional $\mathrm{ZF}$ and SVD methods as well.

\section{B. Bob's Received Signal}

After Alice transmits the signal, the received signal of Bob located at $\left(r_{\mathrm{B}}, \theta_{\mathrm{B}}, \psi_{\mathrm{B}}\right)$ can be written as

$$
\begin{aligned}
y\left(r_{\mathrm{B}}, \theta_{\mathrm{B}}, \psi_{\mathrm{B}}\right) & =\epsilon_{\mathrm{B}} \mathbf{h}_{\mathrm{B}}^{\mathrm{H}} \mathbf{x}+\xi_{\mathrm{B}} \\
& =\epsilon_{\mathrm{B}} \beta_{1} \sqrt{P_{s}} \mathbf{h}_{\mathrm{B}}^{\mathrm{H}} \mathbf{p}_{1} s+\epsilon_{\mathrm{B}} \alpha \beta_{2} \sqrt{P_{s}} \mathbf{h}_{\mathrm{B}}^{\mathrm{H}} \mathbf{p}_{2} z+\xi_{\mathrm{B}} \\
& =\epsilon_{\mathrm{B}} \beta_{1} \sqrt{P_{s}} s+\xi_{\mathrm{B}},
\end{aligned}
$$

where $\xi_{\mathrm{B}}$ is the complex additive white Gaussian noise (AWGN) with zero mean and variance $\delta_{\mathrm{B}}^{2}$, i.e., $\xi_{\mathrm{B}} \sim$ $\mathcal{C N}\left(0, \delta_{\mathrm{B}}^{2}\right)$. In addition, $\epsilon_{\mathrm{B}}$ represents the FTR fading coefficient which is defined as [35]

$$
\epsilon_{\mathrm{B}}=\sqrt{\zeta_{\mathrm{B}}} U_{\mathrm{B}} \exp \left\{\imath \varphi_{\mathrm{B}}\right\}+\sqrt{\zeta_{\mathrm{B}}} V_{\mathrm{B}} \exp \left\{\imath \vartheta_{\mathrm{B}}\right\}+X_{\mathrm{B}}+\imath Y_{\mathrm{B}} \text {, }
$$

where $\zeta_{\mathrm{B}}$ is a Gamma distributed random variable with zero mean and probability density function given by

$$
f_{\zeta_{\mathrm{B}}}\left(\zeta_{\mathrm{B}}\right)=\frac{m_{\mathrm{B}}^{m_{\mathrm{B}}} \zeta_{\mathrm{B}}^{m_{\mathrm{B}}-1}}{\Gamma\left(m_{\mathrm{B}}\right)} \exp \left\{-m_{\mathrm{B}} \zeta_{\mathrm{B}}\right\} .
$$

Moreover, $U_{\mathrm{B}}$ and $V_{\mathrm{B}}$ are constant amplitudes with specular components modulated by a Nakagami- $m_{\mathrm{B}}$ random variable. 


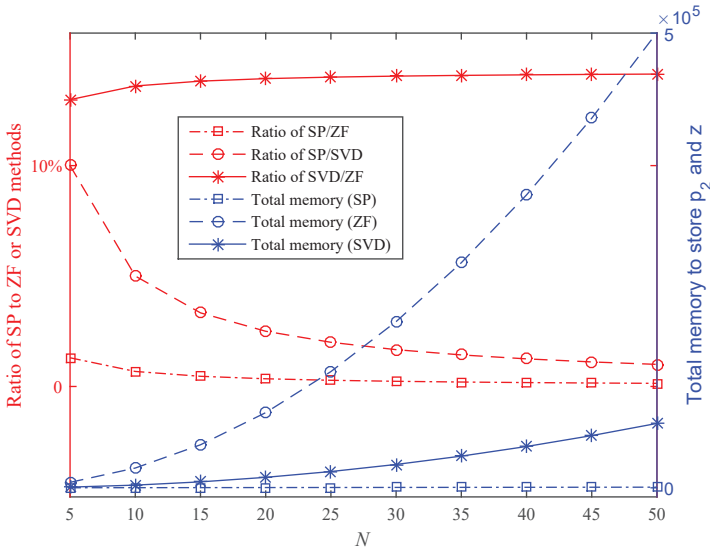

(a)

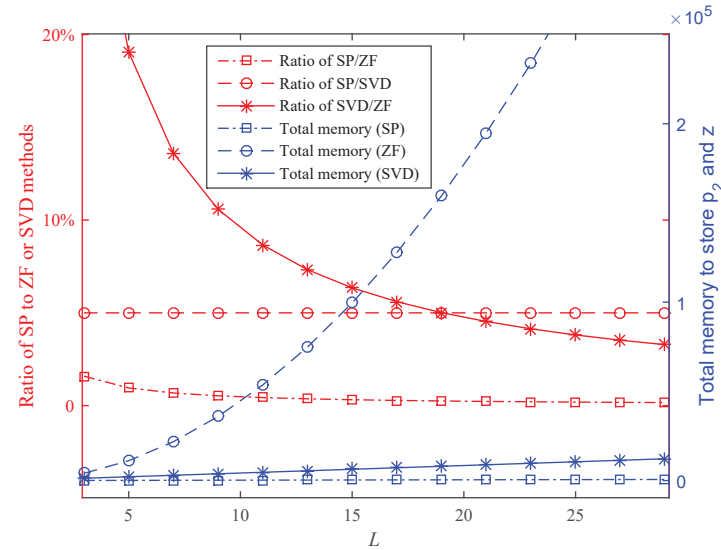

(b)

Fig. 2. Total memory required to store the orthogonal matrix/vector and the AN versus $N$; and the ratio of the proposed SP method to ZF or SVD methods versus (a) $N(L=7)$ and (b) $L(N=10)$.

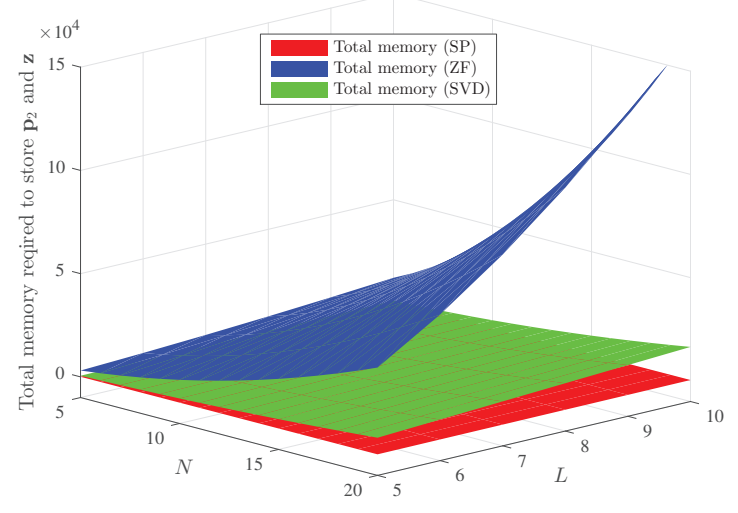

(a)

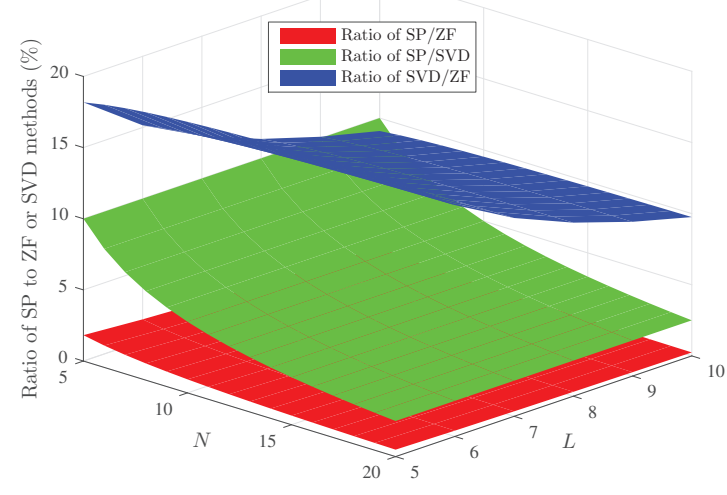

(b)

Fig. 3. (a) Total memory required to store the orthogonal matrix/vector and the AN of the proposed SP method and the conventional ZF and SVD methods and (b) The ratio of total memory of the SP method to that of the ZF or SVD methods versus $N$ and $L$.

$\varphi_{\mathrm{B}}$ and $\vartheta_{\mathrm{B}}$ are statistically independent and uniformly distributed random phases, i.e., $\varphi_{\mathrm{B}}, \vartheta_{\mathrm{B}} \sim \mathcal{U}[0,2 \pi) . X_{\mathrm{B}}+\imath Y_{\mathrm{B}}$ refers to the diffuse component with $X_{\mathrm{B}}$ and $Y_{\mathrm{B}}$ following a Gaussian distribution, i.e., $X_{\mathrm{B}}, Y_{\mathrm{B}} \sim \mathcal{N}\left(0, \sigma_{\mathrm{B}}^{2}\right)$. The FTR fading parameters can be calculated by $K_{\mathrm{B}}=\frac{U_{\mathrm{B}}^{2}+V_{\mathrm{B}}^{2}}{2 \sigma_{\mathrm{B}}^{2}}$ and $\Delta_{\mathrm{B}}=\frac{2 U_{\mathrm{B}} V_{\mathrm{B}}}{U_{\mathrm{B}}^{2}+V_{\mathrm{B}}^{2}}$. It can be observed from (15c) that only the useful signal is left for Bob while the inserted AN has been removed, which guarantees the effective transmission between Alice and Bob.

\section{Eve's Received Signal}

Similarly, the received signal of Eve located at $\left(r_{\mathrm{E}}, \theta_{\mathrm{E}}, \psi_{\mathrm{E}}\right)$, $\left(r_{\mathrm{E}}, \theta_{\mathrm{E}}, \psi_{\mathrm{E}}\right) \neq\left(r_{\mathrm{B}}, \theta_{\mathrm{B}}, \psi_{\mathrm{B}}\right)$, can be expressed as

$$
\begin{aligned}
y\left(r_{\mathrm{E}}, \theta_{\mathrm{E}}, \psi_{\mathrm{E}}\right) & =\epsilon_{\mathrm{E}} \mathbf{h}_{\mathrm{E}}^{\mathrm{H}} \mathbf{x}+\xi_{\mathrm{E}} \\
& =\epsilon_{\mathrm{E}} \beta_{1} \sqrt{P_{s}} \mathbf{h}_{\mathrm{E}}^{\mathrm{H}} \mathbf{p}_{1} s+\epsilon_{\mathrm{E}} \alpha \beta_{2} \sqrt{P_{s}} \mathbf{h}_{\mathrm{E}}^{\mathrm{H}} \mathbf{p}_{2} z+\xi_{\mathrm{E}} \\
& =\underbrace{\epsilon_{\mathrm{E}} \beta_{1} \sqrt{P_{s}} \rho_{1} s}_{\text {Distorted Signal }}+\underbrace{\epsilon_{\mathrm{E}} \alpha \beta_{2} \sqrt{P_{s}} \rho_{2} z}_{\text {Artificial Noise }}+\underbrace{\xi_{\mathrm{E}}}_{\text {AWGN }},
\end{aligned}
$$

where $\xi_{\mathrm{E}} \sim \mathcal{C N}\left(0, \delta_{\mathrm{E}}^{2}\right)$ indicates the complex AWGN, $\mathbf{h}_{\mathrm{E}}=$ $\mathbf{h}\left(r_{\mathrm{E}}, \theta_{\mathrm{E}}, \psi_{\mathrm{E}}\right)$ refers to Eve's normalized steering vector, $\rho_{1}=$ $\mathbf{h}_{\mathrm{E}}^{\mathrm{H}} \mathbf{p}_{1}$, and $\rho_{2}=\mathbf{h}_{\mathrm{E}}^{\mathrm{H}} \mathbf{p}_{2}$. Additionally, the fading coefficient $\epsilon_{\mathrm{E}}$ is defined as

$$
\epsilon_{\mathrm{E}}=\sqrt{\zeta_{\mathrm{E}}} U_{\mathrm{E}} \exp \left\{\imath \varphi_{\mathrm{E}}\right\}+\sqrt{\zeta_{\mathrm{E}}} V_{\mathrm{E}} \exp \left\{\imath \vartheta_{\mathrm{E}}\right\}+X_{\mathrm{E}}+\imath Y_{\mathrm{E}}
$$

which undergoes the FTR fading with the parameters $\left(m_{\mathrm{E}}, K_{\mathrm{E}}, \Delta_{\mathrm{E}}, \sigma_{\mathrm{E}}^{2}\right)$.

It is worth noting that Eve's received signal in (18c) consists of three items. The first is the useful signal distorted by $\rho_{1}$ and the second is the inserted AN, both of which can be regarded as interference for Eve as long as Eve's location differs from Bob's, thereby guaranteeing the PLS transmission between Alice and Bob.

\section{Physical-Layer Security AnAlysis}

In this section, we will analyze the PLS performances of the proposed FDA-DM scheme including the ASC and the SOP, which are important metrics to measure the performances of DM schemes [50]. 


\section{A. PDF And $C D F$}

Before analyzing the ASC and the SOP of the proposed FDA-DM scheme, the probability density functions (PDFs) and cumulative distribution functions (CDFs) of Bob's and Eve's signal-to-inference-plus-noise ratios (SINRs) are first derived in this section.

According to (15c), the received signal of Bob is simply the summation of the useful signal and AWGN, so Bob's SINR can be written as

$$
\gamma_{\mathrm{B}}=\frac{\left|\epsilon_{\mathrm{B}}\right|^{2} \beta_{1}^{2} P_{s} \mathbb{E}\left(|s|^{2}\right)}{\delta_{\mathrm{B}}^{2}}=\beta_{1}^{2} \lambda_{\mathrm{B}},
$$

where $\lambda_{\mathrm{B}}=\left|\epsilon_{\mathrm{B}}\right|^{2} P_{s} \mathbb{E}\left(|s|^{2}\right) / \delta_{\mathrm{B}}^{2}$ is Bob's signal-to-noise ratio (SNR) in the FTR fading channel without splitting $\mathrm{AN}$, the average of which can be calculated by $\bar{\lambda}_{\mathrm{B}}=$ $\mathbb{E}\left(\left|\epsilon_{\mathrm{B}}\right|^{2}\right) P_{s} \mathbb{E}\left(|s|^{2}\right) / \delta_{\mathrm{B}}^{2}=2 \sigma_{\mathrm{B}}^{2}\left(1+K_{\mathrm{B}}\right) P_{s} / \delta_{\mathrm{B}}^{2}[35]$.

Similarly, we can obtain Eve's SINR by observing (18c) as follows,

$$
\gamma_{\mathrm{E}}=\frac{\left|\epsilon_{\mathrm{E}}\right|^{2} \beta_{1}^{2}\left|\rho_{1}\right|^{2} P_{s} \mathbb{E}\left(|s|^{2}\right) / \delta_{\mathrm{E}}^{2}}{\left|\epsilon_{\mathrm{E}}\right|^{2} \alpha^{2} \beta_{2}^{2}\left|\rho_{2}\right|^{2} P_{s} \mathbb{E}\left(|z|^{2}\right) / \delta_{\mathrm{E}}^{2}+1} .
$$

Let $\lambda_{\mathrm{E}}=\left|\epsilon_{\mathrm{E}}\right|^{2} P_{s} \mathbb{E}\left(|s|^{2}\right) / \delta_{\mathrm{E}}^{2}$, which indicates Eve's SNR in the FTR fading channel without splitting AN, the average of which can be calculated by $\bar{\lambda}_{\mathrm{E}}=\mathbb{E}\left(\left|\epsilon_{\mathrm{E}}\right|^{2}\right) P_{s} \mathbb{E}\left(|s|^{2}\right) / \delta_{\mathrm{E}}^{2}=$ $2 \sigma_{\mathrm{E}}^{2}\left(1+K_{\mathrm{E}}\right) P_{s} / \delta_{\mathrm{E}}^{2}$. Moreover, taking the assumption $\mathbb{E}\left(|s|^{2}\right)=\mathbb{E}\left(|z|^{2}\right)=1$ into (21) yields

$$
\begin{aligned}
\gamma_{\mathrm{E}} & =\frac{\beta_{1}^{2}\left|\rho_{1}\right|^{2} \lambda_{\mathrm{E}}}{\alpha^{2} \beta_{2}^{2}\left|\rho_{2}\right|^{2} \lambda_{\mathrm{E}}+1} \\
& =\frac{\eta \lambda_{\mathrm{E}}}{\mu \lambda_{\mathrm{E}}+1},
\end{aligned}
$$

where $\eta=\beta_{1}^{2}\left|\rho_{1}\right|^{2}$ and $\mu=\alpha^{2} \beta_{2}^{2}\left|\rho_{2}\right|^{2}$.

The PDFs and CDFs of the SNR in FTR fading channel without splitting $\mathrm{AN}, \lambda_{i}(i \in\{\mathrm{B}, \mathrm{E}\})$, can be obtained, respectively, by [35]

$$
f_{\lambda_{i}}(x)=\frac{m_{i}^{m_{i}}}{\Gamma\left(m_{i}\right)} \sum_{j_{i}=0}^{\infty} \frac{K_{i}^{j_{i}} d_{j_{i}}}{j_{i} ! j_{i} !} \frac{x^{j_{i}}}{\left(2 \sigma_{i}^{2}\right)^{j_{i}+1}} \exp \left\{-\frac{x}{2 \sigma_{i}^{2}}\right\},
$$

and

$$
F_{\lambda_{i}}(x)=\frac{m_{i}^{m_{i}}}{\Gamma\left(m_{i}\right)} \sum_{j_{i}=0}^{\infty} \frac{K_{i}^{j_{i}} d_{j_{i}}}{j_{i} ! j_{i} !} \Upsilon\left(j_{i}+1, \frac{x}{2 \sigma_{i}^{2}}\right),
$$

where $j_{i}$ ! denotes the factorial of the integer $j_{i} ; \Gamma(\cdot)$ and $\Upsilon(\cdot, \cdot)$ refer to the ordinary Gamma function [51, Eq. (8.310.1)] and the lower incomplete Gamma function [51, Eq. (8.350.1)], respectively. In addition, the term $d_{j_{i}}$ in (23) and (24) is expressed as

$$
\begin{aligned}
d_{j_{i}} \triangleq & \sum_{k=0}^{j_{i}}\left(\begin{array}{c}
j_{i} \\
k
\end{array}\right)\left(\frac{\Delta_{i}}{2}\right)^{k} \sum_{l=0}^{k}\left(\begin{array}{l}
k \\
l
\end{array}\right) \Gamma\left(j_{i}+m_{i}+2 l-k\right) \\
& \times \exp \left\{\imath \frac{(2 l-k) \pi}{2}\right\}\left[\left(m_{i}+K_{i}\right)^{2}-\left(K_{i} \Delta_{i}\right)^{2}\right]^{-\frac{j_{i}+m_{i}}{2}} \\
& \times \mathcal{L}_{j_{i}+m_{i}-1}^{k-2 l}\left(\frac{m_{i}+K_{i}}{\sqrt{\left(m_{i}+K_{i}\right)^{2}-\left(K_{i} \Delta_{i}\right)^{2}}}\right)
\end{aligned}
$$

where $\left(\begin{array}{l}k \\ l\end{array}\right)$ denotes that $k$ chooses $l$, and $\mathcal{L}:(\cdot)$ represents the associated Legendre function of the first kind [51, Eq. (8.702)]. By observing (20), the PDF and CDF of Bob's SINR $\gamma_{\mathrm{B}}$ can be calculated, respectively, by

$$
f_{\gamma_{\mathrm{B}}}(x)=\frac{1}{\beta_{1}^{2}} f_{\lambda_{\mathrm{B}}}\left(\frac{x}{\beta_{1}^{2}}\right),
$$

and

$$
F_{\gamma_{\mathrm{B}}}(x)=F_{\lambda_{\mathrm{B}}}\left(\frac{x}{\beta_{1}^{2}}\right)
$$

Before deriving the PDF and CDF of Eve's SINR $\gamma_{\mathrm{E}}$, it is worth noting that the lower and upper bounds on $\gamma_{\mathrm{E}}$ are 0 and $\tau=\eta / \mu$, respectively, which can be directly obtained by replacing $\lambda_{\mathrm{E}} \rightarrow 0$ and $\lambda_{\mathrm{E}} \rightarrow \infty$ into (22). Therefore, the CDF of $\gamma_{\mathrm{E}}$ can be acquired by

$$
\begin{aligned}
F_{\gamma_{\mathrm{E}}}(x) & =\operatorname{Pr}\left(\gamma_{\mathrm{E}} \leqslant x\right) \\
& =\operatorname{Pr}\left(\frac{\eta \lambda_{\mathrm{E}}}{\mu \lambda_{\mathrm{E}}+1} \leqslant x\right) \\
& =\operatorname{Pr}\left(\lambda_{\mathrm{E}} \leqslant \frac{x}{\eta-\mu x}\right) \\
& =\left\{\begin{array}{cc}
F_{\lambda_{\mathrm{E}}}\left(\frac{x}{\eta-\mu x}\right), & 0<x<\tau \\
1 . & x \geqslant \tau
\end{array}\right.
\end{aligned}
$$

Consequently, the PDF of $\gamma_{\mathrm{E}}$ can be calculated by

$$
\begin{aligned}
f_{\gamma_{\mathrm{E}}}(x) & =\frac{d F_{\gamma_{\mathrm{E}}}(x)}{d x} \\
& =\left\{\begin{array}{cc}
\frac{\eta}{(\eta-\mu x)^{2}} f_{\lambda_{\mathrm{E}}}\left(\frac{x}{\eta-\mu x}\right), & 0<x<\tau \\
0 . & x \geqslant \tau
\end{array}\right.
\end{aligned}
$$

B. $A S C$

For the analysis of ASC, both Bob's and Eve's channel state information (CSI) is assumed to be fully available at Alice, which could be acquired by CSI estimation methods, such as [46], [47]. Practically, it may apply to the scenario where a base station (Alice) serves two independent singleantenna users, who request secure downlink transmissions from Alice. In such a scenario, on the one hand, the users ask for a downlink transmission request from Alice, such that their CSI could be available at Alice; on the other hand, the users do not want the other one to obtain their own private information in case of privacy disclosure, which means they could play dual roles as Bob or Eve for each other. Using the PDFs and CDFs of Bob's and Eve's SINRs in (26)-(29), the instantaneous secrecy capacity can be defined as

$$
C_{s}\left(\gamma_{\mathrm{B}}, \gamma_{\mathrm{E}}\right)=\left[\log _{2}\left(1+\gamma_{\mathrm{B}}\right)-\log _{2}\left(1+\gamma_{\mathrm{E}}\right)\right]^{+},
$$

where $[\cdot]^{+}=\max \{\cdot, 0\}$. If we further assume Bob's and Eve's channels experience independent fading, the ASC can 
be calculated by [38]

$$
\begin{aligned}
\bar{C}_{s}\left(\gamma_{\mathrm{B}}, \gamma_{\mathrm{E}}\right)= & \int_{0}^{\infty} \int_{0}^{\infty} C_{s}\left(\gamma_{\mathrm{B}}, \gamma_{\mathrm{E}}\right) f\left(\gamma_{\mathrm{B}}, \gamma_{\mathrm{E}}\right) d \gamma_{\mathrm{B}} d \gamma_{\mathrm{E}} \\
= & \underbrace{\frac{1}{\ln 2} \int_{0}^{\infty} \ln \left(1+\gamma_{\mathrm{B}}\right) f_{\gamma_{\mathrm{B}}}\left(\gamma_{\mathrm{B}}\right) F_{\gamma_{\mathrm{E}}}\left(\gamma_{\mathrm{B}}\right) d \gamma_{\mathrm{B}}}_{\mathcal{I}_{1}} \\
& +\underbrace{\frac{1}{\ln 2} \int_{0}^{\infty} \ln \left(1+\gamma_{\mathrm{E}}\right) f_{\gamma_{\mathrm{E}}}\left(\gamma_{\mathrm{E}}\right) F_{\gamma_{\mathrm{B}}}\left(\gamma_{\mathrm{E}}\right) d \gamma_{\mathrm{E}}}_{\mathcal{I}_{2}} \\
& -\underbrace{\frac{1}{\ln 2} \int_{0}^{\infty} \ln \left(1+\gamma_{\mathrm{E}}\right) f_{\gamma_{\mathrm{E}}}\left(\gamma_{\mathrm{E}}\right) d \gamma_{\mathrm{E}}}_{\mathcal{I}_{3}}
\end{aligned}
$$

where $f\left(\gamma_{\mathrm{B}}, \gamma_{\mathrm{E}}\right)=f_{\gamma_{\mathrm{B}}}\left(\gamma_{\mathrm{B}}\right) f_{\gamma_{\mathrm{E}}}\left(\gamma_{\mathrm{E}}\right)$ is the joint PDF of $\gamma_{\mathrm{B}}$ and $\gamma_{\mathrm{E}}$.

Before deriving the ASC, we define a function as

$$
\Psi\left(v_{1}, v_{2}, v_{3}, v_{4}, v_{5}, \tau\right)=\int_{0}^{\tau} \mathcal{G}(t) d t
$$

where

$$
\mathcal{G}(t)=\ln ^{v_{1}}(1+t) \frac{t^{v_{2}}}{(\tau-t)^{v_{3}}} \exp \left\{-v_{4} t-\frac{v_{5} t}{\tau-t}\right\} \text {. }
$$

Using standard calculation softwares like MATHEMATICA or MATLAB, (32) can be easily calculated. To provide a closedform insight, an approximated expression for (32) when $\tau<$ $\infty$ can be obtained by applying Gaussian-Legendre quadrature formula [52, Eq. (25.4.30)], i.e.,

$$
\begin{aligned}
\Psi\left(v_{1}, v_{2}, v_{3}, v_{4}, v_{5}, \tau\right) & =\frac{\tau}{2} \int_{-1}^{1} \mathcal{G}\left(\frac{\tau}{2} t+\frac{\tau}{2}\right) d t \\
& \approx \frac{\tau}{2} \sum_{i=1}^{q} \omega_{i} \mathcal{G}\left(\frac{\tau}{2} t_{i}+\frac{\tau}{2}\right),
\end{aligned}
$$

where $t_{i}(i=1,2, \cdots, q)$ is the $i$-th zero point of the $q$-th order Legendre polynomial [51, Eq. (8.910.2)], and $\omega_{i}$ is the $i$-th coefficient [52, Eq. (25.4.29)]. Actually, the approximation in (34) can achieve a perfect agreement with the actual values even with small orders. To illustrate this perfect agreement, we take $\left(v_{1}, v_{2}, v_{3}, v_{4}, v_{5}\right)=(2,4,5,0.5,10.5)$ (Case I) and $\left(v_{1}, v_{2}, v_{3}, v_{4}, v_{5}\right)=(1,3,4,0.6,15.5)$ (Case II) as examples. Fig. 4 shows the comparisons of actual and approximated values by Gauss-Legendre quadrature for the function $\Psi(\tau)$, from which it can be obtained that the Gauss-Legendre quadrature can provide nearly accurate approximation with the actual $\Psi(\tau)$ even with small orders, like $q=10,20$.

Specially, an accurate closed-form expression for (32) when $\tau \rightarrow \infty$ can be written as [30, Eq. (10)]

$$
\begin{aligned}
\mathcal{S}(u, v) & =\Psi(1, u-1,0, v, 0, \infty) \\
& =\int_{0}^{\infty} \ln (1+t) t^{u-1} \exp \{-v t\} d t \\
& =(u-1) ! \exp \{v\} \sum_{k=1}^{u} \frac{\Gamma(-u+k, v)}{v^{k}} .
\end{aligned}
$$

Since the full CSI of both Bob's and Eve's channels is available at Alice, the ASC can be obtained in Lemma 1 by substituting (26), (27), (28d) and (29b) into (31).

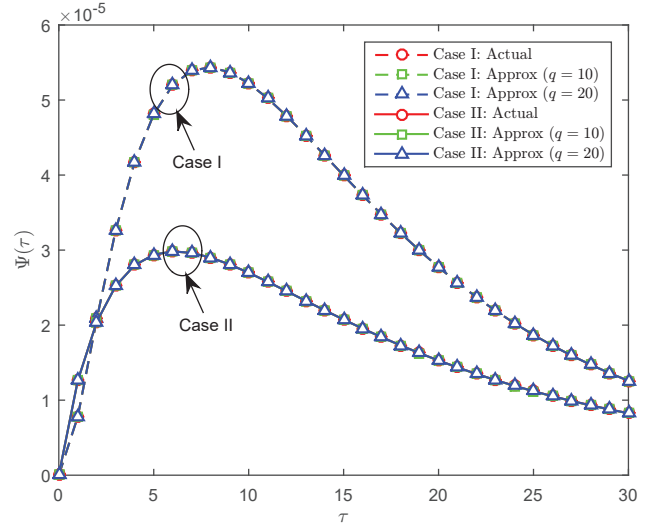

Fig. 4. Comparisons of actual and approximated values by Gauss-Legendre quadrature for $\Psi(\tau)$. Case I: $\left(v_{1}, v_{2}, v_{3}, v_{4}, v_{5}\right)=(2,4,5,0.5,10.5)$, Case II: $\left(v_{1}, v_{2}, v_{3}, v_{4}, v_{5}\right)=(1,3,4,0.6,15.5)$.

Lemma 1: The ASC of the proposed FDA-DM scheme in FTR fading channel is given by

$$
\bar{C}_{s}\left(\gamma_{\mathrm{B}}, \gamma_{\mathrm{E}}\right)=\mathcal{I}_{1}+\mathcal{I}_{2}-\mathcal{I}_{3}
$$

where the expressions of $\mathcal{I}_{1}, \mathcal{I}_{2}$ and $\mathcal{I}_{3}$ are listed in (37), (38) and (39), respectively.

Proof: Please see Appendix A.

Asymptotically, when the diffuse component power $\sigma_{\mathrm{E}}^{2} \rightarrow$ $\infty$, a closed-form expression of the lower bound on the ASC is given in Lemma 2. In fact, the condition $\sigma_{\mathrm{E}}^{2} \rightarrow \infty$ indicates the worst scenario where Eve can achieve the best SINR. Given a fixed FTR fading parameter $K_{\mathrm{E}}=\frac{U_{\mathrm{E}}^{2}+V_{\mathrm{E}}^{2}}{2 \sigma_{\mathrm{E}}^{2}}, \sigma_{\mathrm{E}}^{2} \rightarrow \infty$ implies $U_{\mathrm{E}}^{2}+V_{\mathrm{E}}^{2} \rightarrow \infty$, and further $\left|\epsilon_{\mathrm{E}}\right|^{2} \rightarrow \infty$. According to (21), $\left|\epsilon_{\mathrm{E}}\right|^{2} \rightarrow \infty$ indicates $\lambda_{\mathrm{E}}=\left|\epsilon_{\mathrm{E}}\right|^{2} P_{s} \mathbb{E}\left(|s|^{2}\right) / \delta_{\mathrm{E}}^{2} \rightarrow \infty$, and further $\gamma_{\mathrm{E}} \rightarrow \tau=\eta / \mu$, which is exactly the upper bound on Eve's SINR.

Lemma 2: When $\sigma_{\mathrm{E}}^{2} \rightarrow \infty$, the lower bound on the ASC of the proposed FDA-DM scheme in FTR fading can be written in the following closed-form expression,

$$
\begin{aligned}
\bar{C}_{s}^{\text {Low }}\left(\gamma_{\mathrm{B}}, \gamma_{\mathrm{E}}\right) \\
=\frac{m_{\mathrm{B}}^{m_{\mathrm{B}}}}{\ln 2 \Gamma\left(m_{\mathrm{B}}\right)} \sum_{j_{\mathrm{B}}=0}^{\infty} \frac{K_{\mathrm{B}}^{j_{\mathrm{B}}} d_{j_{\mathrm{B}}}}{j_{\mathrm{B}} ! j_{\mathrm{B}} !\left(2 \beta_{1}^{2} \sigma_{\mathrm{B}}^{2}\right)^{j_{\mathrm{B}}+1}} \\
\quad \times\left[\mathcal{S}\left(j_{\mathrm{B}}+1, \frac{1}{2 \beta_{1}^{2} \sigma_{\mathrm{B}}^{2}}\right)-\Psi\left(1, j_{\mathrm{B}}, 0, \frac{1}{2 \beta_{1}^{2} \sigma_{\mathrm{B}}^{2}}, 0, \tau\right)\right] \\
\quad-\frac{\ln (1+\tau)}{\ln 2}\left[1-F_{\gamma_{\mathrm{B}}}(\tau)\right] .
\end{aligned}
$$

Proof: Please see Appendix B.

\section{SOP}

Different from ASC, the calculation of SOP does not require Alice to obtain Eve's CSI, which means Alice has to encode the confidential information at a certain rate $R_{0}$. From the information-theoretic perspective, the perfect secrecy can be achieved if $R_{0} \leq C_{s}$; otherwise, the secrecy outage will occur. 
The SOP is defined as the probability that the instantaneous secrecy capacity $C_{s}$ falls below a target secrecy rate $R_{0}$, i.e.,

$$
\begin{aligned}
P_{\text {out }} & =\operatorname{Pr}\left\{C_{s}\left(\gamma_{\mathrm{B}}, \gamma_{\mathrm{E}}\right)<R_{0}\right\} \\
& =\operatorname{Pr}\left\{\log _{2} \frac{1+\gamma_{\mathrm{B}}}{1+\gamma_{\mathrm{E}}}<R_{0}\right\},
\end{aligned}
$$

which can be mathematically calculated in Lemma 3 .

Lemma 3: The SOP of the proposed FDA-DM scheme in FTR fading can be obtained by

$$
\begin{aligned}
P_{\mathrm{out}}= & \frac{m_{\mathrm{B}}^{m_{\mathrm{B}}}}{\Gamma\left(m_{\mathrm{B}}\right)} \frac{m_{\mathrm{E}}^{m_{\mathrm{E}}}}{\Gamma\left(m_{\mathrm{E}}\right)} \sum_{j_{\mathrm{B}}=0}^{\infty} \sum_{j_{\mathrm{E}}=0}^{\infty} \frac{K_{\mathrm{B}}^{j_{\mathrm{B}}} d_{j_{\mathrm{B}}} K_{\mathrm{E}}^{j_{\mathrm{E}}} d_{j_{\mathrm{E}}} \tau}{j_{\mathrm{B}} ! j_{\mathrm{E}} ! j_{\mathrm{E}} !\left(2 \mu \sigma_{\mathrm{E}}^{2}\right)^{j_{\mathrm{E}}+1}} \\
& \times\left[\Psi\left(0, j_{\mathrm{E}}, j_{\mathrm{E}}+2,0, \frac{1}{2 \mu \sigma_{\mathrm{E}}^{2}}, \tau\right)\right. \\
& -\sum_{n=0}^{j_{\mathrm{B}}} \frac{1}{n !} \exp \left(-\frac{2^{R_{0}}-1}{2 \beta_{1}^{2} \sigma_{\mathrm{B}}^{2}}\right) \sum_{k=0}^{n}\left(\begin{array}{l}
n \\
k
\end{array}\right) \frac{2^{k R_{0}}\left(2^{R_{0}}-1\right)^{n-k}}{\left(2 \beta_{1}^{2} \sigma_{\mathrm{B}}^{2}\right)^{n}} \\
& \left.\times \Psi\left(0, j_{\mathrm{E}}+k, j_{\mathrm{E}}+2, \frac{2^{R_{0}}}{2 \beta_{1}^{2} \sigma_{\mathrm{B}}^{2}}, \frac{1}{2 \mu \sigma_{\mathrm{E}}^{2}}, \tau\right)\right] .
\end{aligned}
$$

Proof: Please see Appendix C.

Lemma 4: When $\sigma_{\mathrm{E}}^{2} \rightarrow \infty$, the upper bound on the SOP of the proposed FDA-DM scheme in FTR fading can be written as

$$
P_{\text {out }}^{\mathrm{Up}}=\frac{m_{\mathrm{B}}^{m_{\mathrm{B}}}}{\Gamma\left(m_{\mathrm{B}}\right)} \sum_{j_{\mathrm{B}}=0}^{\infty} \frac{K_{\mathrm{B}}^{j_{\mathrm{B}}} d_{j_{\mathrm{B}}}}{j_{\mathrm{B}} ! j_{\mathrm{B}} !} \Upsilon\left(j_{\mathrm{B}}+1, \frac{2^{R_{0}}(1+\tau)-1}{2 \beta_{1}^{2} \sigma_{\mathrm{B}}^{2}}\right) .
$$

Proof: Please see Appendix D.

\section{Numerical Results}

In this section, Monte Carlo experiments are conducted to verify the theoretical analysis, where the ZF method [16], [18], [21], [27]-[32], the SVD method [22], and the NoAN method [38]-[40] are included. The detailed parameters are listed in
TABLE II

SiMUlation PARAMETERS

\begin{tabular}{ll}
\hline Parameter & Value \\
\hline Central frequency, $f_{0}$ & $30 \mathrm{GHz}$ \\
Fixed frequency increment, $\Delta f$ & $20 \mathrm{kHz}$ \\
Number of FDA elements, $2 N+1$ & 21 \\
Number of subcarriers for each element, $L$ & 7 \\
Total transmit power, $P_{s}$ & $30 \mathrm{dBm}$ \\
Power splitting factor, $\beta_{1}$ & 0.9 \\
AWGN variance, $\delta_{\mathrm{B}}^{2}, \delta_{\mathrm{E}}^{2}$ & 1 \\
FTR parameters, $\left(m_{\mathrm{B}}, K_{\mathrm{B}}, \Delta_{\mathrm{B}}\right)$ & $(2.3,10,0.5)$ \\
FTR parameters, $\left(m_{\mathrm{E}}, K_{\mathrm{E}}, \Delta_{\mathrm{E}}\right)$ & $(5.3,15,0.35)$ \\
Location of Bob, $\left(r_{\mathrm{B}}, \theta_{\mathrm{B}}, \psi_{\mathrm{B}}\right)$ & $\left(1 \mathrm{~km}, 20^{\circ}, 30^{\circ}\right)$ \\
Location of Eve, $\left(r_{\mathrm{E}}, \theta_{\mathrm{E}}, \psi_{\mathrm{E}}\right)$ & $\left(1.5 \mathrm{~km},-20^{\circ}, 25^{\circ}\right)$ \\
Number of Monte Carlo experiments & $10^{5}$ \\
\hline
\end{tabular}

Table II. For fair consideration, the AWGN powers $\delta_{\mathrm{B}}^{2}$ and $\delta_{\mathrm{E}}^{2}$ are set as identical fixed values, and the total transmit power $P_{s}$ is set as a fixed value as well, which means Alice-Bob and Alice-Eve AWGN channels have the same quality.

\section{A. $A S C$}

Fig. 5(a) depicts the ASC of the proposed FDA-DM scheme versus $\bar{\lambda}_{\mathrm{B}}$ in FTR fading channels, where the analytical results well match the simulated results with $10^{5}$ Monte Carlo experiments. First, it holds for all four PLS approaches that the ASC climbs with increasing $\bar{\lambda}_{\mathrm{B}}$ when $\bar{\lambda}_{\mathrm{E}}$ is fixed. Second, compared with ZF and SVD approaches, the proposed SP method can achieve almost the same ASC. Although there is actually a very small penalty on ASC, the proposed SP method is still much more competitive considering the considerable memory savings. Moreover, compared with the NoAN approach, there is a positive lower bound on the ASC when $\bar{\lambda}_{\mathrm{E}} \rightarrow \infty$, which means an absolutely positive ASC can always been realized

$$
\begin{aligned}
& \mathcal{I}_{1}= \frac{m_{\mathrm{B}}^{m_{\mathrm{B}}} m_{\mathrm{E}}^{m_{\mathrm{E}}}}{\ln 2 \Gamma\left(m_{\mathrm{B}}\right) \Gamma\left(m_{\mathrm{E}}\right)} \sum_{j_{\mathrm{B}}=0}^{\infty} \sum_{j_{\mathrm{E}}=0}^{\infty} \frac{K_{\mathrm{B}}^{j_{\mathrm{B}}} d_{j_{\mathrm{B}}} K_{\mathrm{E}}^{j_{\mathrm{E}}} d_{j_{\mathrm{E}}}}{j_{\mathrm{B}} ! j_{\mathrm{B}} ! j_{\mathrm{E}} !\left(2 \beta_{1}^{2} \sigma_{\mathrm{B}}^{2}\right)^{j_{\mathrm{B}}+1}} \\
& \times\left(\Psi\left(1, j_{\mathrm{B}}, 0, \frac{1}{2 \beta_{1}^{2} \sigma_{\mathrm{B}}^{2}}, 0, \tau\right)-\sum_{n=0}^{j_{\mathrm{E}}} \frac{1}{n !\left(2 \mu \sigma_{\mathrm{E}}^{2}\right)^{n}} \Psi\left(1, j_{\mathrm{B}}+n, n, \frac{1}{2 \beta_{1}^{2} \sigma_{\mathrm{B}}^{2}}, \frac{1}{2 \mu \sigma_{\mathrm{E}}^{2}}, \tau\right)\right) \\
&+\frac{m_{\mathrm{B}}^{m_{\mathrm{B}}}}{\ln 2 \Gamma\left(m_{\mathrm{B}}\right)} \sum_{j_{\mathrm{B}}=0}^{\infty} \frac{K_{\mathrm{B}}^{j_{\mathrm{B}}} d_{j_{\mathrm{B}}}}{j_{\mathrm{B}} ! j_{\mathrm{B}} !\left(2 \beta_{1}^{2} \sigma_{\mathrm{B}}^{2}\right)^{j_{\mathrm{B}}+1}}\left(\mathcal{S}\left(j_{\mathrm{B}}+1, \frac{1}{2 \beta_{1}^{2} \sigma_{\mathrm{B}}^{2}}\right)-\Psi\left(1, j_{\mathrm{B}}, 0, \frac{1}{2 \beta_{1}^{2} \sigma_{\mathrm{B}}^{2}}, 0, \tau\right)\right) . \\
& \mathcal{I}_{2}= \frac{m_{\mathrm{B}}^{m_{\mathrm{B}}} m_{\mathrm{E}}^{m_{\mathrm{E}}}}{\ln 2 \Gamma\left(m_{\mathrm{B}}\right) \Gamma\left(m_{\mathrm{E}}\right)} \sum_{j_{\mathrm{B}}=0}^{\infty} \sum_{j_{\mathrm{E}}=0}^{\infty} \frac{K_{\mathrm{B}}^{j_{\mathrm{B}}} d_{j_{\mathrm{B}}} K_{\mathrm{E}}^{j_{\mathrm{E}}} d_{j_{\mathrm{E}}} \tau}{j_{\mathrm{E}} ! j_{\mathrm{E}} !\left(2 \mu \sigma_{\mathrm{E}}^{2}\right)^{j_{\mathrm{E}}+1}} \\
&\left(\Psi\left(1, j_{\mathrm{E}}, j_{\mathrm{E}}+2,0, \frac{1}{2 \mu \sigma_{\mathrm{E}}^{2}}, \tau\right)-\sum_{n=0}^{j_{\mathrm{B}}} \frac{1}{n !\left(2 \beta_{1}^{2} \sigma_{\mathrm{B}}^{2}\right)^{n}} \Psi\left(1, j_{\mathrm{E}}+n, j_{\mathrm{E}}+2, \frac{1}{2 \beta_{1}^{2} \sigma_{\mathrm{B}}^{2}}, \frac{1}{2 \mu \sigma_{\mathrm{E}}^{2}}, \tau\right)\right) . \\
& \mathcal{I}_{3}=\frac{m_{\mathrm{E}}^{m_{\mathrm{E}}}}{\ln 2 \Gamma\left(m_{\mathrm{E}}\right)} \sum_{j_{\mathrm{E}}=0}^{\infty} \frac{K_{\mathrm{E}}^{j_{\mathrm{E}}} d_{j_{\mathrm{E}}} \tau}{j_{\mathrm{E}} ! j_{\mathrm{E}} !\left(2 \mu \sigma_{\mathrm{E}}^{2}\right)^{j_{\mathrm{E}}+1}} \Psi\left(1, j_{\mathrm{E}}, j_{\mathrm{E}}+2,0, \frac{1}{2 \mu \sigma_{\mathrm{E}}^{2}}, \tau\right) .
\end{aligned}
$$




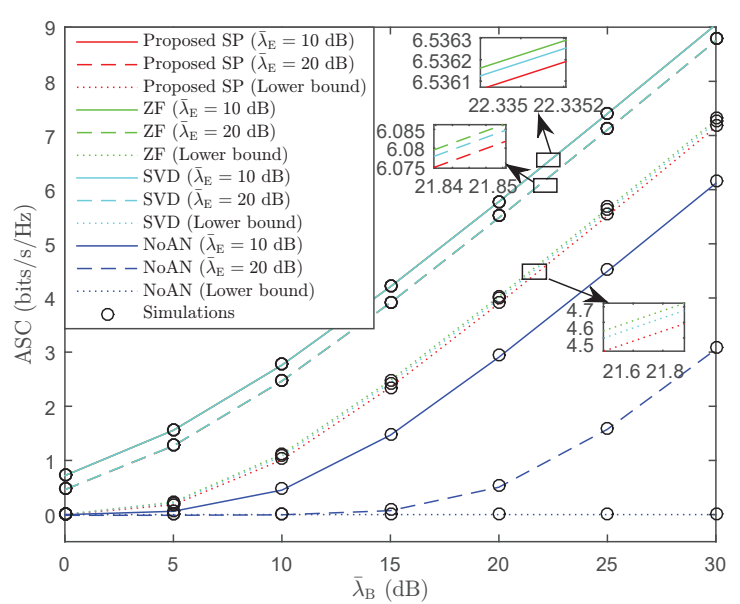

(a)

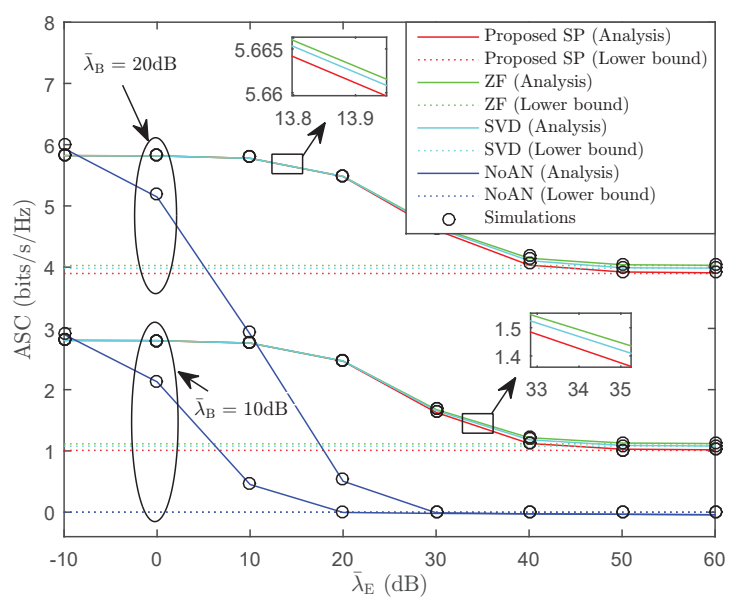

(b)

Fig. 5. ASC $\bar{C}_{s}$ versus (a) Bob's and (b) Eve's average SNRs $\bar{\lambda}_{\mathrm{B}}(\mathrm{dB})$ and $\bar{\lambda}_{\mathrm{E}}(\mathrm{dB})$ in FTR fading channels. $\left(f_{0}=30 \mathrm{GHz}, \Delta f=20 \mathrm{kHz}, N=10\right.$, $L=7, \beta_{1}=0.9$ )

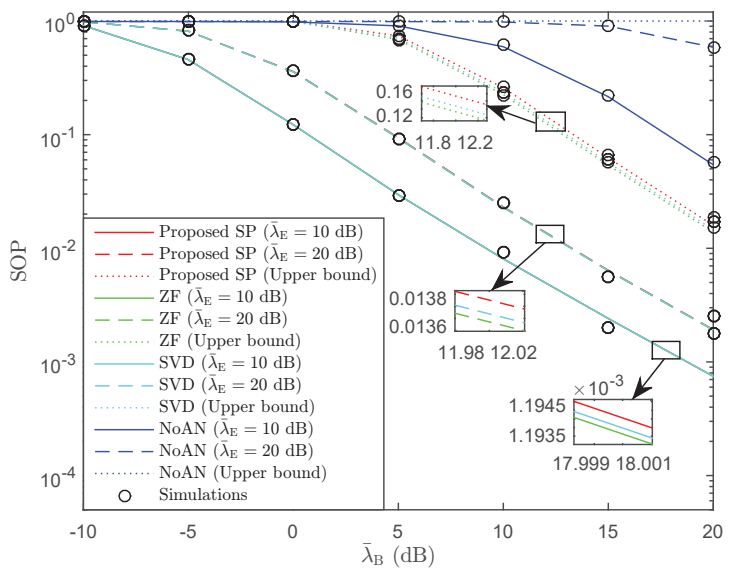

(a)

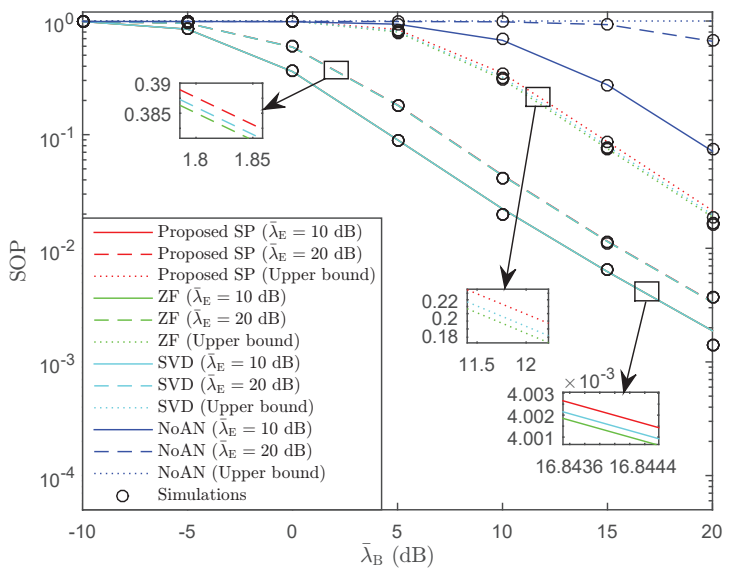

(b)

Fig. 6. SOP $P_{\text {out }}$ versus Bob's average SNR $\bar{\lambda}_{\mathrm{B}}(\mathrm{dB})$ with (a) $R_{0}=0.2$ bits $/ \mathrm{s} / \mathrm{Hz}$ and (b) $R_{0}=0.5$ bits/s/Hz in FTR fading channels. $\left(f_{0}=30\right.$ GHz, $\left.\Delta f=20 \mathrm{kHz}, N=10, L=7, \beta_{1}=0.9\right)$

as long as $\bar{\lambda}_{\mathrm{B}}$ is big enough. By contrast, the ASC of NoAN approach reduces to zero when $\bar{\lambda}_{\mathrm{E}} \rightarrow \infty$.

Fig. 5(b) illustrates the ASC of the proposed FDA-DM scheme versus $\bar{\lambda}_{\mathrm{E}}$ in FTR fading channels. As expected, that the ASC decreases with increasing $\bar{\lambda}_{\mathrm{E}}$ when $\bar{\lambda}_{\mathrm{B}}$ is fixed. Differently, the ASCs of the proposed SP and the conventional ZF and SVD methods reduce to a positive lower bound, while the ASC of the NoAN method declines to zero. Similar to Fig. 5(a), almost the same ASC can be achieved for the proposed SP and the conventional ZF and SVD methods while the proposed SP distinguishes from the conventional ZF and SVD methods with much lower memory consumption as analyzed in Section III.A.

\section{B. $S O P$}

Figs. 6(a) and (b) present the SOP of the proposed FDADM scheme versus $\bar{\lambda}_{\mathrm{B}}$ in FTR fading channels with $R_{0}=0.2$ and 0.5 bits $/ \mathrm{s} / \mathrm{Hz}$, respectively. Comparing Figs. 6(a) and (b), it holds for all four PLS methods that the SOP decreases with smaller $R_{0}$. Given a specific $R_{0}$ and $\bar{\lambda}_{\mathrm{E}}$, as expected, the
SOP drops as $\bar{\lambda}_{\mathrm{B}}$ increases. Compared with the conventional NoAN method, the proposed SP method can achieve much lower SOP. More importantly, there exists an upper bound on SOP when $\bar{\lambda}_{\mathrm{E}} \rightarrow \infty$ for the proposed SP method, while the SOP of the conventional NoAN method roars to 1 . On the other hand, the proposed SP method can achieve almost the same SOP as the conventional ZF and SVD approaches with much lower memory consumption.

Figs. 7(a) and (b) illustrate the SOP of the proposed FDADM scheme versus $\bar{\lambda}_{\mathrm{E}}$ in FTR fading channels with $R_{0}=0.2$ and $0.5 \mathrm{bits} / \mathrm{s} / \mathrm{Hz}$, respectively. Analogous to Figs. 6(a) and (b), it can also be observed that a smaller $R_{0}$ produces a smaller SOP. With a specific $R_{0}$, the SOPs of the proposed SP method and the conventional ZF and SVD methods increase to an upper bound along with increasing $\bar{\lambda}_{\mathrm{E}}$. But for the conventional NoAN method, it roars rapidly to 1 when $\bar{\lambda}_{\mathrm{E}}$ increases. The advantage of the proposed SP method is verified again that much lower memory consumption yet achieves almost the same SOP as the ZF and SVD methods. 


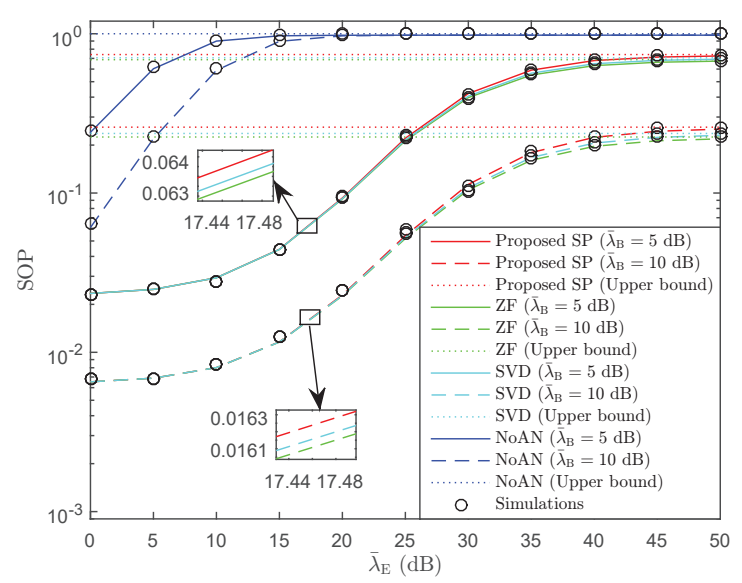

(a)

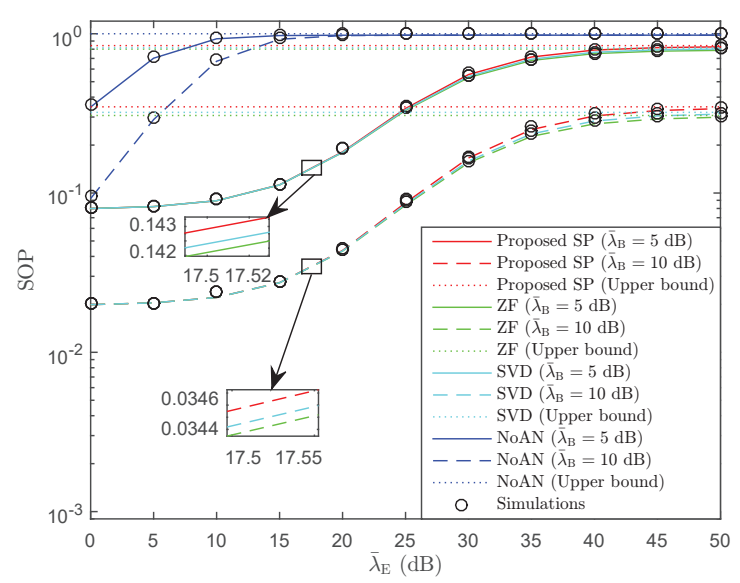

(b)

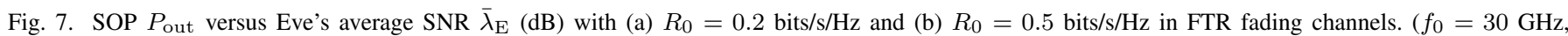
$\left.\Delta f=20 \mathrm{kHz}, N=10, L=7, \beta_{1}=0.9\right)$

TABLE III

Improved or Lost Performances of The Proposed SP Method Compared with ZF AND SVD Methods $\left(f_{0}=30\right.$ GHz, $\Delta f=20 \mathrm{kHz}$, $N=10, L=7, \beta_{1}=0.9, \bar{\lambda}_{\mathrm{B}}=20 \mathrm{~dB}, \bar{\lambda}_{\mathrm{E}}=10 \mathrm{~dB}$, AND $\left.R_{0}=0.2 \mathrm{bits} / \mathrm{s} / \mathrm{Hz}\right)$

\begin{tabular}{|c|c|c|c|c|c|}
\hline Items & $\mathbf{Z F}[16],[18],[21],[27]-[32]$ & SVD [22] & Proposed SP & $\frac{|\mathrm{ZF}-\mathrm{SP}|}{\mathrm{ZF}} \times 100 \%$ & $\frac{|\mathrm{SVD}-\mathrm{SP}|}{\mathrm{SVD}} \times 100 \%$ \\
\hline Memory Consumption & 21756 & 2960 & 148 & $99.32 \%$ (Improved) & $95 \%$ (Improved) \\
\hline $\mathrm{ASC}, \bar{C}_{s}(\mathrm{bits} / \mathrm{s} / \mathrm{Hz})$ & 5.77768102 & 5.77764192 & 5.77757348 & $0.0018 \%$ (Lost) & $0.0012 \%$ (Lost) \\
\hline SOP, $P_{\text {out }}\left(\times 10^{-4}\right)$ & 7.47269793 & 7.47377494 & 7.47559205 & $0.0387 \%$ (Lost) & $0.0243 \%$ (Lost) \\
\hline
\end{tabular}

\section{Benefit and Detriment}

In order to demonstrate the improvement or loss of performances, we take $f_{0}=30 \mathrm{GHz}, \Delta f=20 \mathrm{kHz}, N=10$, $L=7, \beta_{1}=0.9, \bar{\lambda}_{\mathrm{B}}=20 \mathrm{~dB}, \bar{\lambda}_{\mathrm{E}}=10 \mathrm{~dB}$, and $R_{0}=0.2 \mathrm{bits} / \mathrm{s} / \mathrm{Hz}$ as an example. Table III illustrates the improved or lost performances of the proposed SP method compared with conventional ZF and SVD methods, where the total memory consumption, the ASC and the SOP are presented. We can obtain that compared with the ZF (or SVD) method, the proposed SP method can save $99.32 \%$ (or $95 \%$ ) total memory while only cost $0.0018 \%$ (or $0.0012 \%$ ) loss of the ASC, and $0.0387 \%$ (or $0.0243 \%$ ) loss of the SOP. Therefore, for the proposed SP method, the benefit of significant memory savings outweighs the detriment of very tiny secrecy degradation.

\section{Discussions on a Multi-Antenna Eve}

In this section, the secrecy performance with a multiantenna Eve is discussed. We assume the multi-antenna Eve is equipped with $J$ antennas, and the channel coefficient vector from Alice to Eve's $J$ antennas is denoted by $\epsilon_{\mathrm{E}}=$ $\left[\begin{array}{lllll}\epsilon_{\mathrm{E}, 1} & \cdots & \epsilon_{\mathrm{E}, j} & \cdots & \epsilon_{\mathrm{E}, J}\end{array}\right]^{\mathrm{T}}$, of which the $j$-th entry $(j=$ $1,2, \cdots, J), \epsilon_{\mathrm{E}, j}$, undergoes FTR fading with parameters $\left(m_{\mathrm{E}, j}, K_{\mathrm{E}, j}, \Delta_{\mathrm{E}, j}, \sigma_{\mathrm{E}, j}^{2}\right)$. Then, the received signal vector of the multi-antenna (MA) Eve located at $\left(r_{\mathrm{E}}, \theta_{\mathrm{E}}, \psi_{\mathrm{E}}\right)$ can be obtained by

$$
\begin{aligned}
\mathbf{y}^{\mathrm{MA}}\left(r_{\mathrm{E}}, \theta_{\mathrm{E}}, \psi_{\mathrm{E}}\right) & =\boldsymbol{\epsilon}_{\mathrm{E}} \mathbf{h}_{\mathrm{E}}^{\mathrm{H}} \mathbf{x}+\boldsymbol{\xi}_{\mathrm{E}} \\
= & \boldsymbol{\epsilon}_{\mathrm{E}} \beta_{1} \sqrt{P_{s}} \mathbf{h}_{\mathrm{E}}^{\mathrm{H}} \mathbf{p}_{1} s+\boldsymbol{\epsilon}_{\mathrm{E}} \alpha \beta_{2} \sqrt{P_{s}} \mathbf{h}_{\mathrm{E}}^{\mathrm{H}} \mathbf{p}_{2} z+\boldsymbol{\xi}_{\mathrm{E}} \\
= & \underbrace{\boldsymbol{\epsilon}_{\mathrm{E}} \beta_{1} \sqrt{P_{s}} \rho_{1} s}_{\text {Distorted Signal }}+\underbrace{\boldsymbol{\epsilon}_{\mathrm{E}} \alpha \beta_{2} \sqrt{P_{s}} \rho_{2} z}_{\text {Artificial Noise }}+\underbrace{\boldsymbol{\xi}_{\mathrm{E}}}_{\text {AWGN }},
\end{aligned}
$$

where $\boldsymbol{\xi}_{\mathrm{E}}=\left[\begin{array}{lllll}\xi_{\mathrm{E}, 1} & \cdots & \xi_{\mathrm{E}, j} & \cdots & \xi_{\mathrm{E}, J}\end{array}\right]^{\mathrm{T}} \sim \mathcal{C N}\left(\mathbf{0}, \delta_{\mathrm{E}}^{2} \mathbf{I}_{J}\right)$ indicates Eve's complex AWGN vector. According to (44c), the SINR of the multi-antenna Eve can be rewritten as

$$
\begin{aligned}
\gamma_{\mathrm{E}}^{\mathrm{MA}} & =\frac{\left\|\boldsymbol{\epsilon}_{\mathrm{E}}\right\|^{2} \beta_{1}^{2}\left|\rho_{1}\right|^{2} P_{s} \mathbb{E}\left(|s|^{2}\right) / \delta_{\mathrm{E}}^{2}}{\|\left.\boldsymbol{\epsilon}_{\mathrm{E}}\right|^{2} \alpha^{2} \beta_{2}^{2}\left|\rho_{2}\right|^{2} P_{s} \mathbb{E}\left(|z|^{2}\right) / \delta_{\mathrm{E}}^{2}+1} \\
& =\frac{\eta \lambda_{\mathrm{E}}^{\mathrm{MA}}}{\mu \lambda_{\mathrm{E}}^{\mathrm{MA}}+1},
\end{aligned}
$$

where $\lambda_{\mathrm{E}}^{\mathrm{MA}}=\left\|\boldsymbol{\epsilon}_{\mathrm{E}}\right\|^{2} P_{s} \mathbb{E}\left(|s|^{2}\right) / \delta_{\mathrm{E}}^{2}$ represents the multiantenna Eve's SNR. It is easy to observe that $\lambda_{\mathrm{E}}^{\mathrm{MA}}$ is a sum of squared FTR random variables, of which the PDF and CDF can be calculated by [37, Eqs.(5) and (6)].

According to above analysis, the simulated ASCs with single-antenna $(J=1)$ and multi-antenna $(J=7,100, \infty)$ Eves are shown in Fig. 8. It can be observed from Fig. 8 that 1) no matter Eve has single or multiple antenna(s), the proposed SP method can achieve almost the same ASC as the conventional ZF and SVD methods; 2) compared with the conventional NoAN method, only a slight ASC reduction occurs for the proposed SP method when Eve has multiple antennas, while the ASC of the NoAN method reduces dramatically with a multi-antenna Eve; 3) the ASC has a lower bound when 


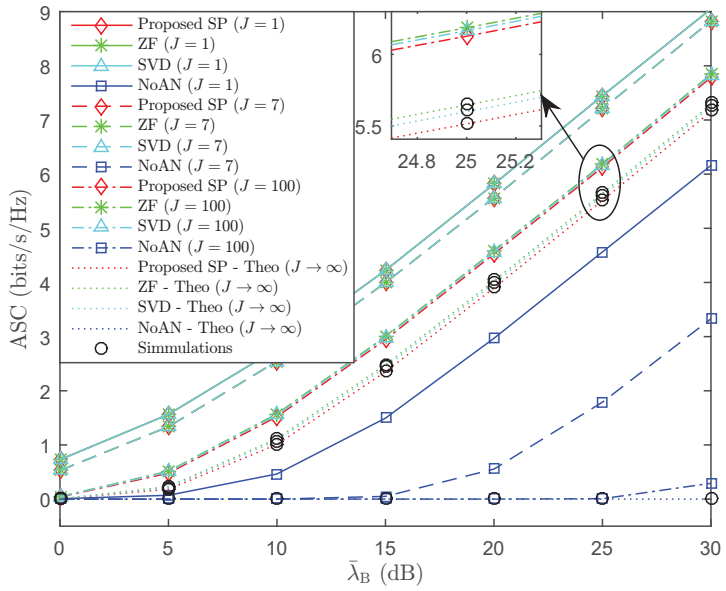

(a)

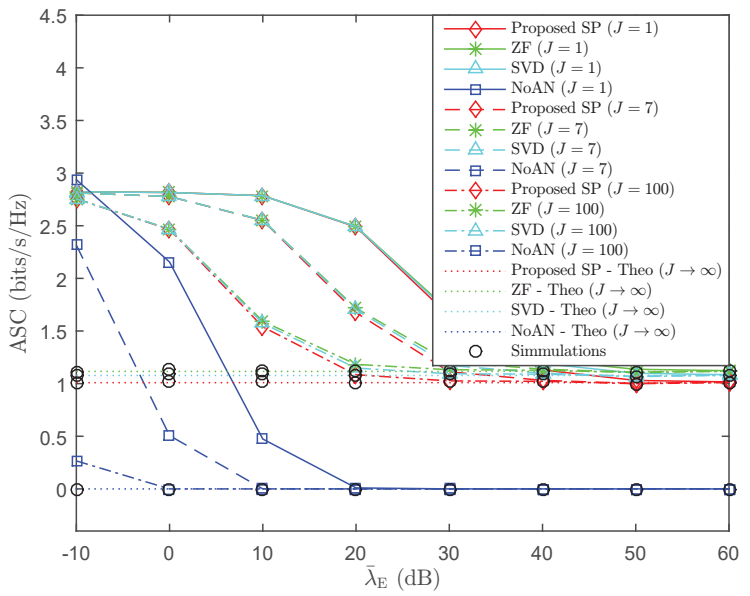

(b)

Fig. 8. ASC $\bar{C}_{s}$ versus (a) Bob's average SNR $\bar{\lambda}_{\mathrm{B}}\left(\bar{\lambda}_{\mathrm{E}}=10 \mathrm{~dB}\right)$ and (b) Eve's average SNR $\bar{\lambda}_{\mathrm{E}}\left(\bar{\lambda}_{\mathrm{B}}=10 \mathrm{~dB}\right)$ in FTR fading channels with a multi-antenna Eve. $\left(f_{0}=30 \mathrm{GHz}, \Delta f=20 \mathrm{kHz}, N=10, L=7, \beta_{1}=0.9\right)$

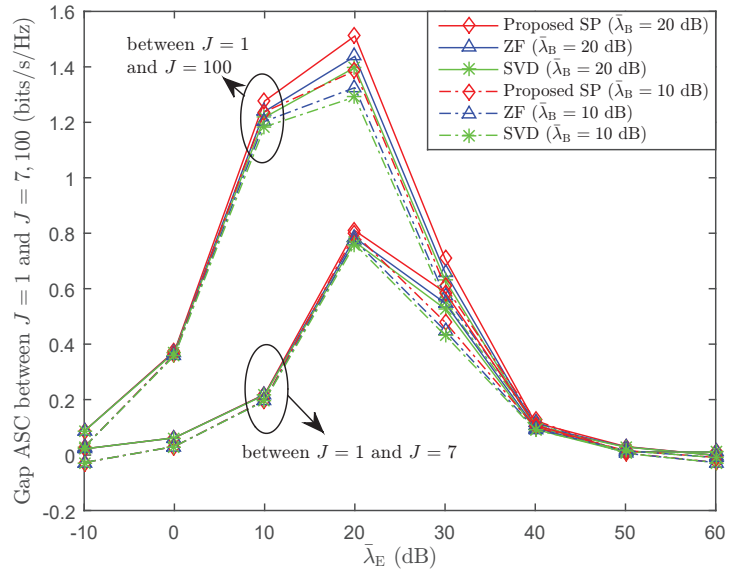

Fig. 9. Gap ASCs between single-antenna Eve $(J=1)$ and multi-antenna Eve $(J=7,100)$. $\left(f_{0}=30 \mathrm{GHz}, \Delta f=20 \mathrm{kHz}, N=10, L=7\right.$, $\left.\beta_{1}=0.9\right)$

Eve's antenna number $J$ goes to $\infty$, which is the same as the single-antenna case. As a matter of fact, $J \rightarrow \infty$ implies $\lambda_{\mathrm{E}}^{\mathrm{MA}} \rightarrow \infty$ and further $\gamma_{\mathrm{E}}^{\mathrm{MA}} \rightarrow \tau=\eta / \mu$, which accounts for the lower bound on ASC.

In order to further illustrate the secrecy degradation, we depict the gap ASC between the single-antenna $(J=1)$ and multi-antenna $(J=7,100)$ Eves in Fig. 9. It shows that along with the increase of Eve's average SNR, the gap ASC (secrecy degradation) climbs from zero to a peak value before reducing to zero again. This means that a) the multi-antenna $(J>1)$ Eve cannot degrade the secrecy performance when its average SNR $\bar{\lambda}_{\mathrm{E}}$ is sufficiently small or large; b) the secrecy performance will be slightly degraded only when $\bar{\lambda}_{\mathrm{E}}$ falls into a "middle" range (for example, between $0 \mathrm{~dB}$ and $40 \mathrm{~dB}$ with the given simulation parameters). As a matter of fact, this phenomenon can be explained by (22) and (45). On the one hand, from (22), the lower and upper bounds on $\bar{\gamma}_{\mathrm{E}}$ (singleantenna) are $\bar{\gamma}_{\mathrm{E}}^{\text {Low }}=0$ and $\bar{\gamma}_{\mathrm{E}}^{\mathrm{Up}}=\tau=\eta / \mu$. On the other hand, it can be obtained from (45) that $\bar{\lambda}_{\mathrm{E}}^{\mathrm{MA}} \rightarrow 0$ and $\infty$ correspond to the lower and upper bounds on $\bar{\gamma}_{\mathrm{E}}^{\mathrm{MA}}$ (multi- antenna), i.e., $\bar{\gamma}_{\mathrm{E}}^{\mathrm{MA}, \mathrm{Low}}=0$ and $\bar{\gamma}_{\mathrm{E}}^{\mathrm{MA}, \mathrm{Up}}=\tau=\eta / \mu$, which are the same as the case with a single-antenna Eve. Therefore, when the average SNR $\bar{\lambda}_{\mathrm{E}}$ is sufficiently small or large, the secrecy performance will not be noticeably degraded even with a multi-antenna Eve.

\section{E. Discussions on the Time-Variance of FDA}

Owing to the small frequency increments between adjacent antennas, the far-field beampattern radiated by FDA is timevariant, which is a common problem for nearly all types of FDA including the multi-carrier FDA utilized in this paper. The time-variant problem may result in short-time secure transmissions via the proposed FDA-DM scheme, which may be possibly used for secure signaling control from a base station to legitimate users.

To achieve long-time secure transmissions via the proposed FDA-DM scheme, the time-variant problem of FDA should be properly addressed, which, however, is not the focus of this paper. As a matter of fact, the key purposes of this paper are to present a memory-efficient SP method and to analyze the PLS performances of DM systems in FTR fading channels. Although only the multi-carrier FDA model is adopted in this paper, the proposed SP method and the PLS analysis on ASC and SOP in FTR fading channels do not depend on some specific antenna array, which are flexible and applicable to other antenna arrays (like PA)-based DM schemes as well.

\section{CONCLUSION}

In this paper, we presented a memory-efficient single-point AN-aided secure DM transmission scheme for $5 \mathrm{G}$ and beyond communications based on symmetrical multi-carrier FDA, which significantly outperforms the conventional ZF and SVD approaches with only a very small penalty on secrecy. In the proposed FDA-DM scheme, the FDA was analyzed in three dimensions, i.e., range, azimuth angle, and elevation angle. Moreover, the ASC and SOP of the proposed FDADM scheme were analyzed, for the first time, in FTR fading channels, which provide a better fit for small-scale fading 
measurements in mmWave communications. The closed-form expressions of lower ASC bound and upper SOP bound were derived and numerical demonstrations with Monte Carlo trials were provided as well. This work opens a way to lower the memory requirements for DM-based PLS transmission of $5 \mathrm{G}$ and beyond communications. Some challenges and future works are to investigate the PLS performance of the proposed FDA-DM scheme with multiple multi-antenna users and to solve the time-variant problem of FDA.

\section{APPENDIX}

\section{A. Proof of Lemma 1}

Here, we derive the expression of $\mathcal{I}_{1}$. Substituting (26) and (28d) into (31), we can obtain (46), where $\Upsilon\left(j_{\mathrm{E}}+1, \frac{\gamma_{\mathrm{B}}}{2 \sigma_{\mathrm{E}}^{2}\left(\eta-\mu \gamma_{\mathrm{B}}\right)}\right)$ can be written as 47 [51, Eq. (8.354.1)]. Substituting (47) into (46b) and using (32), we can acquire

$$
\begin{aligned}
\mathcal{I}_{1,1}= & j_{\mathrm{E}} ! \Psi\left(1, j_{\mathrm{B}}, 0, \frac{1}{2 \beta_{1}^{2} \sigma_{\mathrm{B}}^{2}}, 0, \tau\right) \\
& -j_{\mathrm{E}} ! \sum_{n=0}^{j_{\mathrm{E}}} \frac{1}{n !\left(2 \mu \sigma_{\mathrm{E}}^{2}\right)^{n}} \Psi\left(1, j_{\mathrm{B}}+n, n, \frac{1}{2 \beta_{1}^{2} \sigma_{\mathrm{B}}^{2}}, \frac{1}{2 \mu \sigma_{\mathrm{E}}^{2}}, \tau\right) .
\end{aligned}
$$

Similarly, $\mathcal{I}_{1,2}$ can be calculated by

$$
\mathcal{I}_{1,2}=\Psi\left(1, j_{\mathrm{B}}, 0, \frac{1}{2 \beta_{1}^{2} \sigma_{\mathrm{B}}^{2}}, 0, \infty\right)-\Psi\left(1, j_{\mathrm{B}}, 0, \frac{1}{2 \beta_{1}^{2} \sigma_{\mathrm{B}}^{2}}, 0, \tau\right) .
$$

Substituting (48) and (49) into (46b), we can get the expression of $\mathcal{I}_{1}$ in (37). $\mathcal{I}_{2}$ and $\mathcal{I}_{3}$ can be derived in the same way, which ends the proof of Lemma 1.

\section{B. Proof of Lemma 2}

Observing (22), when $\sigma_{\mathrm{E}}^{2} \rightarrow \infty$, we can subsequently obtain $\lambda_{\mathrm{E}} \rightarrow \infty$ and $\gamma_{\mathrm{E}} \rightarrow \tau$. Therefore, $\bar{C}_{s}^{\text {Low }}$ can be written as

$$
\begin{aligned}
\bar{C}_{s}^{\text {Low }}\left(\gamma_{\mathrm{B}}, \gamma_{\mathrm{E}}\right) \\
=\frac{1}{\ln 2} \int_{0}^{\infty}\left[\ln \left(1+\gamma_{\mathrm{B}}\right)-\ln (1+\tau)\right]^{+} f_{\gamma_{\mathrm{B}}}\left(\gamma_{\mathrm{B}}\right) d \gamma_{\mathrm{B}} \\
=\frac{1}{\ln 2} \int_{\tau}^{\infty} \ln \left(1+\gamma_{\mathrm{B}}\right) f_{\gamma_{\mathrm{B}}}\left(\gamma_{\mathrm{B}}\right) d \gamma_{\mathrm{B}} \\
\quad-\frac{1}{\ln 2} \int_{\tau}^{\infty} \ln (1+\tau) f_{\gamma_{\mathrm{B}}}\left(\gamma_{\mathrm{B}}\right) d \gamma_{\mathrm{B}} .
\end{aligned}
$$

Replacing (23) and (26) into (50) yields (51), and substituting (32) into (51b) ends the proof of Lemma 2.

\section{Proof of Lemma 3}

Given a fixed secrecy capacity $R_{0}$, the SOP in (41b) can be further written as

$$
\begin{aligned}
P_{\text {out }} & =\operatorname{Pr}\left\{\gamma_{\mathrm{B}}<2^{R_{0}}\left(1+\gamma_{\mathrm{E}}\right)-1\right\} \\
& =\int_{0}^{\infty} F_{\gamma_{\mathrm{B}}}\left(2^{R_{0}}\left(1+\gamma_{\mathrm{E}}\right)-1\right) f_{\gamma_{\mathrm{E}}}\left(\gamma_{\mathrm{E}}\right) d \gamma_{\mathrm{E}} .
\end{aligned}
$$

Then, replacing (23), (26) and (29b) into (52b) yields (53). Using [51, Eq. (8.354.1)], the term $\mathcal{A}$ in (53b) can be calculated by (54), where

$$
\begin{aligned}
\mathcal{B}= & \int_{0}^{\tau} \frac{\gamma_{\mathrm{E}}^{j_{\mathrm{E}}}}{\left(\tau-\gamma_{\mathrm{E}}\right)^{j_{\mathrm{E}}+2}}\left(\frac{2^{R_{0}}\left(1+\gamma_{\mathrm{E}}\right)-1}{2 \beta_{1}^{2} \sigma_{\mathrm{B}}^{2}}\right)^{n} \\
& \times \exp \left(-\frac{\gamma_{\mathrm{E}}}{2 \mu \sigma_{\mathrm{E}}^{2}\left(\tau-\gamma_{\mathrm{E}}\right)}-\frac{2^{R_{0}}\left(1+\gamma_{\mathrm{E}}\right)-1}{2 \beta_{1}^{2} \sigma_{\mathrm{B}}^{2}}\right) d \gamma_{\mathrm{E}} .
\end{aligned}
$$

According to the binomial theorem [51, Eq. (1.111)], we can write

$$
\begin{gathered}
\left(\frac{2^{R_{0}}\left(1+\gamma_{\mathrm{E}}\right)-1}{2 \beta_{1}^{2} \sigma_{\mathrm{B}}^{2}}\right)^{n}=\left(\frac{2^{R_{0}}}{2 \beta_{1}^{2} \sigma_{\mathrm{B}}^{2}} \gamma_{\mathrm{E}}+\frac{2^{R_{0}}-1}{2 \beta_{1}^{2} \sigma_{\mathrm{B}}^{2}}\right)^{n} \\
=\frac{2^{n R_{0}}}{\left(2 \beta_{1}^{2} \sigma_{\mathrm{B}}^{2}\right)^{n}} \sum_{k=0}^{n}\left(\begin{array}{l}
n \\
k
\end{array}\right) \gamma_{\mathrm{E}}^{k}\left(\frac{2^{R_{0}}-1}{2^{R_{0}}}\right)^{n-k}
\end{gathered}
$$

$$
\begin{aligned}
& \mathcal{I}_{1}= \frac{1}{\ln 2} \int_{0}^{\tau} \ln \left(1+\gamma_{\mathrm{B}}\right) f_{\gamma_{\mathrm{B}}}\left(\gamma_{\mathrm{B}}\right) F_{\gamma_{\mathrm{E}}}\left(\gamma_{\mathrm{B}}\right) d \gamma_{\mathrm{B}}+\frac{1}{\ln 2} \int_{\tau}^{\infty} \ln \left(1+\gamma_{\mathrm{B}}\right) f_{\gamma_{\mathrm{B}}}\left(\gamma_{\mathrm{B}}\right) F_{\gamma_{\mathrm{E}}}\left(\gamma_{\mathrm{B}}\right) d \gamma_{\mathrm{B}} \\
&= \frac{m_{\mathrm{B}}^{m_{\mathrm{B}}} m_{\mathrm{E}}^{m_{\mathrm{E}}}}{\ln 2 \Gamma\left(m_{\mathrm{B}}\right) \Gamma\left(m_{\mathrm{E}}\right)} \sum_{j_{\mathrm{B}}=0}^{\infty} \sum_{j_{\mathrm{E}}=0}^{\infty} \frac{K_{\mathrm{B}}^{j_{\mathrm{B}}} d_{j_{\mathrm{B}}} K_{\mathrm{E}}^{j_{\mathrm{E}}} d_{j_{\mathrm{E}}}}{j_{\mathrm{B}} ! j_{\mathrm{B}} ! j_{\mathrm{E}} ! j_{\mathrm{E}} !\left(2 \beta_{1}^{2} \sigma_{\mathrm{B}}^{2}\right)^{j_{\mathrm{B}}+1}} \\
& \times \underbrace{\int_{0}^{\tau} \ln \left(1+\gamma_{\mathrm{B}}\right) \gamma_{\mathrm{B}}^{j_{\mathrm{B}}} \exp \left(-\frac{\gamma_{\mathrm{B}}}{2 \beta_{1}^{2} \sigma_{\mathrm{B}}^{2}}\right) \Upsilon\left(j_{\mathrm{E}}+1, \frac{\gamma_{\mathrm{B}}}{2 \sigma_{\mathrm{E}}^{2}\left(\eta-\mu \gamma_{\mathrm{B}}\right)}\right) d \gamma_{\mathrm{B}}}_{\mathcal{I}_{1,1}} \\
&+\frac{m_{\mathrm{B}}^{m_{\mathrm{B}}}}{\ln 2 \Gamma\left(m_{\mathrm{B}}\right)} \sum_{j_{\mathrm{B}}=0}^{\infty} \frac{K_{\mathrm{B}}^{j_{\mathrm{B}}} d_{j_{\mathrm{B}}}}{j_{\mathrm{B}} ! j_{\mathrm{B}} !\left(2 \beta_{1}^{2} \sigma_{\mathrm{B}}^{2}\right)^{j_{\mathrm{B}}+1}} \underbrace{\int_{\tau}^{\infty} \ln \left(1+\gamma_{\mathrm{B}}\right) \gamma_{\mathrm{B}}^{j_{\mathrm{B}}} \exp \left(-\frac{\gamma_{\mathrm{B}}}{2 \beta_{1}^{2} \sigma_{\mathrm{B}}^{2}}\right) d \gamma_{\mathrm{B}}}_{\tau} . \\
& \Upsilon\left(j_{\mathrm{E}}+1, \frac{\gamma_{\mathrm{B}}}{2 \sigma_{\mathrm{E}}^{2}\left(\eta-\mu \gamma_{\mathrm{B}}\right)}\right)=j_{\mathrm{E}} !\left(1-\exp \left(-\frac{\gamma_{\mathrm{B}}}{2 \sigma_{\mathrm{E}}^{2}\left(\eta-\mu \gamma_{\mathrm{B}}\right)}\right) \sum_{n=0}^{j_{\mathrm{E}}} \frac{1}{n !}\left(\frac{\gamma_{\mathrm{B}}}{2 \sigma_{\mathrm{E}}^{2}\left(\eta-\mu \gamma_{\mathrm{B}}\right)}\right)^{n}\right) .
\end{aligned}
$$


Therefore, $\mathcal{B}$ can be further rewritten in (57) by replacing (56b) into (55). Finally, substituting (54c) and (57c) into (53b) ends the proof of Lemma 3.

\section{Proof of Lemma 4}

According to the definition of SOP, the upper bound on SOP when $\sigma_{\mathrm{E}}^{2} \rightarrow \infty$ can be written as

$$
\begin{aligned}
P_{\text {out }}^{\mathrm{Up}} & =\left.\operatorname{Pr}\left\{\log _{2} \frac{1+\gamma_{\mathrm{B}}}{1+\gamma_{\mathrm{E}}}<R_{0}\right\}\right|_{\sigma_{\mathrm{E}}^{2} \rightarrow \infty} \\
& =\operatorname{Pr}\left\{\gamma_{\mathrm{B}}<2^{R_{0}}(1+\tau)-1\right\} \\
& =F_{\gamma_{\mathrm{B}}}\left(2^{R_{0}}(1+\tau)-1\right) .
\end{aligned}
$$

Then applying (27) into (58c), we can obtain

$$
P_{\text {out }}^{\mathrm{Up}}=\frac{m_{\mathrm{B}}^{m_{\mathrm{B}}}}{\Gamma\left(m_{\mathrm{B}}\right)} \sum_{j_{\mathrm{B}}=0}^{\infty} \frac{K_{\mathrm{B}}^{j_{\mathrm{B}}} d_{j_{\mathrm{B}}}}{j_{\mathrm{B}} ! j_{\mathrm{B}} !} \Upsilon\left(j_{\mathrm{B}}+1, \frac{2^{R_{0}}(1+\tau)-1}{2 \beta_{1}^{2} \sigma_{\mathrm{B}}^{2}}\right),
$$

which ends the proof of Lemma 4.

\section{ACKNOWLEDGMENT}

The authors would like to thank Dr. Jiakang Zheng and Prof. Jiayi Zhang at Beijing Jiaotong University for their constructive suggestions about the theoretical PDF calculation of the FTR fading channel.

\section{REFERENCES}

[1] Y. Zou, J. Zhu, X. Wang, and L. Hanzo, "A survey on wireless security: Technical challenges, recent advances, and future trends," Proc. IEEE, vol. 104, no. 9, pp. 1727-1765, Sep. 2016.

[2] Y. Liu, H.-H. Chen, and L. Wang, "Physical layer security for next generation wireless networks: Theories, technologies, and challenges," IEEE Commun. Surveys Tuts., vol. 19, no. 1, pp. 347-376, 1st Quart., 2017.

[3] J. M. Hamamreh, H. M. Furqan, and H. Arslan, "Classifications and applications of physical layer security techniques for confidentiality: A comprehensive survey," IEEE Commun. Surveys Tuts., vol. 21, no. 2, pp. 1773-1828, 2nd Quart., 2018.

[4] N. Yang, S. Yan, J. Yuan, R. Malaney, R. Subramanian, and I. Land, "Artificial noise: Transmission optimization in multi-input single-output wiretap channels," IEEE Trans. Commun., vol. 63, no. 5, pp. 1771-1783, May 2015.

$$
\begin{aligned}
\bar{C}_{s}^{\text {Low }}\left(\gamma_{\mathrm{B}}, \gamma_{\mathrm{E}}\right) & =\frac{1}{\ln 2} \int_{\tau}^{\infty} \ln \left(1+\gamma_{\mathrm{B}}\right) \frac{m_{\mathrm{B}}^{m_{\mathrm{B}}}}{\Gamma\left(m_{\mathrm{B}}\right)} \sum_{j_{\mathrm{B}}=0}^{\infty} \frac{K_{\mathrm{B}}^{j_{\mathrm{B}}} d_{j_{\mathrm{B}}}}{j_{\mathrm{B}} ! j_{\mathrm{B}} !} \frac{\gamma_{\mathrm{B}}^{j_{\mathrm{B}}}}{\left(2 \beta_{1}^{2} \sigma_{\mathrm{B}}^{2}\right)^{j_{\mathrm{B}}+1}} \exp \left(-\frac{\gamma_{\mathrm{B}}}{2 \beta_{1}^{2} \sigma_{\mathrm{B}}^{2}}\right) d \gamma_{\mathrm{B}}-\frac{\ln (1+\tau)}{\ln 2}\left[1-F_{\gamma_{\mathrm{B}}}(\tau)\right] \\
& =\frac{m_{\mathrm{B}}^{m_{\mathrm{B}}}}{\ln 2 \Gamma\left(m_{\mathrm{B}}\right)} \sum_{j_{\mathrm{B}}=0}^{\infty} \frac{K_{\mathrm{B}}^{j_{\mathrm{B}}} d_{j_{\mathrm{B}}}}{j_{\mathrm{B}} ! j_{\mathrm{B}} !\left(2 \beta_{1}^{2} \sigma_{\mathrm{B}}^{2}\right)^{j_{\mathrm{B}}+1}} \int_{\tau}^{\infty} \ln \left(1+\gamma_{\mathrm{B}}\right) \gamma_{\mathrm{B}}^{j_{\mathrm{B}}} \exp \left(-\frac{\gamma_{\mathrm{B}}}{2 \beta_{1}^{2} \sigma_{\mathrm{B}}^{2}}\right) d \gamma_{\mathrm{B}}-\frac{\ln (1+\tau)}{\ln 2}\left[1-F_{\gamma_{\mathrm{B}}}(\tau)\right]
\end{aligned}
$$

$$
\begin{aligned}
P_{\text {out }}= & \int_{0}^{\tau} \frac{m_{\mathrm{B}}^{m_{\mathrm{B}}}}{\Gamma\left(m_{\mathrm{B}}\right)} \sum_{j_{\mathrm{B}}=0}^{\infty} \frac{K_{\mathrm{B}}^{j_{\mathrm{B}}} d_{j_{\mathrm{B}}}}{j_{\mathrm{B}} ! j_{\mathrm{B}} !} \Upsilon\left(j_{\mathrm{B}}+1, \frac{2^{R_{0}}\left(1+\gamma_{\mathrm{E}}\right)-1}{2 \beta_{1}^{2} \sigma_{\mathrm{B}}^{2}}\right) \frac{\eta}{\left(\eta-\mu \gamma_{\mathrm{E}}\right)^{2}} \frac{m_{\mathrm{E}}^{m_{\mathrm{E}}}}{\Gamma\left(m_{\mathrm{E}}\right)} \\
& \times \sum_{j_{\mathrm{E}}=0}^{\infty} \frac{K_{\mathrm{E}}^{j_{\mathrm{E}}} d_{j_{\mathrm{E}}}}{j_{\mathrm{E}} ! j_{\mathrm{E}} !} \frac{\left(\frac{\gamma_{\mathrm{E}}}{\eta-\mu \gamma_{\mathrm{E}}}\right)^{j_{\mathrm{E}}}}{\left(2 \sigma_{\mathrm{E}}^{2}\right)^{j_{\mathrm{E}}+1}} \exp \left(-\frac{\gamma_{\mathrm{E}}}{\eta-\mu \gamma_{\mathrm{E}}}\right) d \gamma_{\mathrm{E}} \\
= & \frac{m_{\mathrm{B}}^{m_{\mathrm{B}}}}{\Gamma\left(m_{\mathrm{B}}\right)} \frac{m_{\mathrm{E}}^{m_{\mathrm{E}}}}{\Gamma\left(m_{\mathrm{E}}\right)} \sum_{j_{\mathrm{B}}=0}^{\infty} \sum_{j_{\mathrm{E}}=0}^{\infty} \frac{K_{\mathrm{B}}^{j_{\mathrm{B}}} d_{j_{\mathrm{B}}} K_{\mathrm{E}}^{j_{\mathrm{E}}} d_{j_{\mathrm{E}}} \tau}{j_{\mathrm{B}} ! j_{\mathrm{B}} ! j_{\mathrm{E}} ! j_{\mathrm{E}} !\left(2 \mu \sigma_{\mathrm{E}}^{2}\right)^{j_{\mathrm{E}}+1}} \\
& \times \underbrace{\int_{0}^{\tau} \Upsilon\left(j_{\mathrm{B}}+1, \frac{2^{R_{0}}\left(1+\gamma_{\mathrm{E}}\right)-1}{2 \beta_{1}^{2} \sigma_{\mathrm{B}}^{2}}\right) \frac{\gamma_{\mathrm{E}}^{j_{\mathrm{E}}}}{\left(\tau-\gamma_{\mathrm{E}}\right)^{j_{\mathrm{E}}+2}} \exp \left(-\frac{\gamma_{\mathrm{E}}}{2 \mu \sigma_{\mathrm{E}}^{2}\left(\tau-\gamma_{\mathrm{E}}\right)}\right) d \gamma_{\mathrm{E}}}_{\mathcal{A}} .
\end{aligned}
$$

$$
\begin{aligned}
\mathcal{A}= & \int_{0}^{\tau} j_{\mathrm{B}} !\left(1-\exp \left(-\frac{2^{R_{0}}\left(1+\gamma_{\mathrm{E}}\right)-1}{2 \beta_{1}^{2} \sigma_{\mathrm{B}}^{2}}\right) \sum_{n=0}^{j_{\mathrm{B}}} \frac{1}{n !}\left(\frac{2^{R_{0}}\left(1+\gamma_{\mathrm{E}}\right)-1}{2 \beta_{1}^{2} \sigma_{\mathrm{B}}^{2}}\right)^{n}\right) \\
& \times \frac{\gamma_{\mathrm{E}}^{j_{\mathrm{E}}}}{\left(\tau-\gamma_{\mathrm{E}}\right)^{j_{\mathrm{E}}+2}} \exp \left(-\frac{\gamma_{\mathrm{E}}}{2 \mu \sigma_{\mathrm{E}}^{2}\left(\tau-\gamma_{\mathrm{E}}\right)}\right) d \gamma_{\mathrm{E}} \\
= & j_{\mathrm{B}} ! \int_{0}^{\tau} \frac{\gamma_{\mathrm{E}}^{j_{\mathrm{E}}}}{\left(\tau-\gamma_{\mathrm{E}}\right)^{j_{\mathrm{E}}+2}} \exp \left(-\frac{\gamma_{\mathrm{E}}}{2 \mu \sigma_{\mathrm{E}}^{2}\left(\tau-\gamma_{\mathrm{E}}\right)}\right) d \gamma_{\mathrm{E}}-\sum_{n=0}^{j_{\mathrm{B}}} \frac{j_{\mathrm{B}} !}{n !} \mathcal{B} \\
= & j_{\mathrm{B}} ! \Psi\left(0, j_{\mathrm{E}}, j_{\mathrm{E}}+2,0, \frac{1}{2 \mu \sigma_{\mathrm{E}}^{2}}, \tau\right)-\sum_{n=0}^{j_{\mathrm{B}}} \frac{j_{\mathrm{B}} !}{n !} \mathcal{B} .
\end{aligned}
$$


[5] X. Chen, D. W. K. Ng, W. H. Gerstacker, and H.-H. Chen, "A survey on multiple-antenna techniques for physical layer security," IEEE Commun. Surveys Tuts., vol. 19, no. 2, pp. 1027-1053, 2nd Quart., 2017.

[6] N. Yang, L. Wang, G. Geraci, M. Elkashlan, J. Yuan, and M. D. Renzo, "Safeguarding 5G wireless communication networks using physical layer security," IEEE Commun. Magaz., vol. 53, no. 4, pp. 20-27, Apr. 2015.

[7] Y. Wu, A. Khisti, C. Xiao, G. Caire, K.-K. Wong, and X. Gao, "A survey of physical layer security techniques for $5 \mathrm{G}$ wireless networks and challenges ahead," IEEE J. Sel. Areas Commun., vol. 36, no. 4, pp. 679-695, Apr. 2018.

[8] Y. Ding and V. Fusco, "A review of directional modulation technology," Int. J. Microw. Wirel. Tech., vol. 8, no. 7, pp. 981-993, Nov. 2016.

[9] W.-Q. Wang and Z. Zheng, "Hybrid MIMO and phased-array directional modulation for physical layer security in mmWave wireless communications," IEEE J. Sel. Areas Commun., vol. 36, no. 7, pp. 1383-1396, Jul. 2018.

[10] S. Y. Nusenu, "Development of frequency modulated array antennas for millimeter-wave communications," Wirel. Commun. \& Mobile Computing, vol. 2019, pp. 1-16, Apr. 2019.

[11] M. P. Daly and J. T. Bernhard, "Directional modulation technique for phased arrays," IEEE Trans. Ante. \& Propag., vol. 57, no. 9, pp. 26332640, Sep. 2009.

[12] W.-Q. Wang, "Frequency diverse array antenna: new opportunities," IEEE Ante. \& Propag. Magaz., vol. 57, no. 2, pp. 145-152, Apr. 2015.

[13] S. Y. Nusenu and A. Basit, "Frequency diverse array antennas: from their origin to their application in wireless communication systems," $J$. Computer Netw. \& Commun., vol. 2018, pp. 1-12, May 2018.

[14] J. Xiong, S. Y. Nusenu, and W.-Q. Wang, "Directional modulation using frequency diverse array for secure communications," Wirel. Personal Commun., vol. 95, no. 3, pp. 2679-2689, Aug. 2017.

[15] W.-Q. Wang, "DM using FDA antenna for secure transmission," IET Microw., Ante. \& Propag., vol. 11, no. 3, pp. 336-345, Apr. 2017.

[16] J. Hu, S. Yan, F. Shu, J. Wang, J. Li, and Y. Zhang, "Artificial-noiseaided secure transmission with directional modulation based on random frequency diverse arrays," IEEE Access, vol. 5, no. 99, pp. 1658-1667, Jan. 2017.

[17] Q. Cheng, J. Zhu, T. Xie, J. Luo, and Z. Xu, "Time-invariant angle-range dependent directional modulation based on time-modulated frequency diverse arrays," IEEE Access, vol. 5, pp. 26279-26290, Dec. 2017.

[18] B. Qiu, J. Xie, L. Wang, and Y. Wang, "Artificial-noise-aided secure transmission for proximal legitimate user and eavesdropper based on frequency diverse arrays," IEEE Access, vol. 6, pp. 52531-52543, 2018.

[19] J. Lin, Q. Li, J. Yang, H. Shao, and W.-Q. Wang, "Physical-layer security for proximal legitimate user and eavesdropper: a frequency diverse array beamforming approach," IEEE Trans. Inform. Forens. \& Sec., vol. 13, no. 3, pp. 671-684, Mar. 2018.

[20] T. Xie, J. Zhu, Q. Cheng, and Y. Guan, "Secure point-to-multipoint communication using the spread spectrum assisted orthogonal frequency diverse array in free space," IEICE Trans. Commun., vol. E102.B, no. 6, pp. 1188-1197, Jun. 2019

[21] B. Qiu, M. Tao, L. Wang, J. Xie, and Y. Wang, "Multi-beam directional modulation synthesis scheme based on frequency diverse array," IEEE Trans. Info. Foren. \& Sec., vol. 14, no. 10, pp. 2593-2606, Oct. 2019.

[22] Q. Cheng, V. Fusco, J. Zhu, S. Wang, and C. Gu, "SVD-aided multibeam directional modulation scheme based on frequency diverse array," IEEE Wirel. Commun. Lett., vol. 9, no. 3, pp. 420-423, Mar. 2020.

[23] Q. Cheng, V. Fusco, J. Zhu, S. Wang, and F. Wang, "WFRFT-aided power-efficient multi-beam directional modulation schemes based on frequency diverse array," IEEE Trans. Wirel. Commun., vol. 18, no. 11, pp. 5211-5226, Nov. 2019.

[24] B. Qiu, L. Wang, J. Xie, Z. Zhang, Y. Wang, and M. Tao, "Multi-beam index modulation with cooperative legitimate users schemes based on frequency diverse array," Accepted by IEEE Trans. Veh. Tech., to appear, DOI:10.1109/TVT.2020.3007003.

[25] T. Shen, S. Zhang, R. Chen, J. Wang, J, Hu, F. Shu, and J. Wang, "Two practical random-subcarrier-selection methods for secure precise wireless transmissions," IEEE Trans. Veh. Techn., vol. 68, no. 9, pp. 9018-9028, Sept. 2019.

[26] F. Shu, X. Wu, J. Hu, J. Li, R. Chen, and J. Wang, "Secure and precise wireless transmission for random-subcarrier-selection-based directional modulation transmit antenna array," IEEE J. Sel. Areas Commun., vol. 36, no. 4, pp. 890-904, Apr. 2018.

[27] J. Hu, F. Shu, and J. Li, "Robust synthesis method for secure directional modulation with imperfect direction angle," IEEE Commun. Lett., vol. 20, no. 6, pp. 1084-1087, Jun. 2016.

[28] F. Shu, L. Xu, J. Wang, W. Zhu, and X. Zhou, "Artificial-noise-aided secure multicast precoding for directional modulation systems," IEEE Trans. Veh. Tech., vol. 67, no. 7, pp. 6658-6662, Jul. 2018.

[29] T. Xie, J. Zhu, and Y. Li, "Artificial-noise-aided zero-forcing synthesis approach for secure multi-beam directional modulation," IEEE Commun. Lett., vol. 22, no. 2, pp. 276-279, Feb. 2018.

[30] S. Ji, W.-Q. Wang, H. Chen, and S. Zhang, "On physical-layer security of FDA communications over Rayleigh fading channels," IEEE Trans. Cogn. Commun. and Net., vol. 5, no. 3, pp. 476-490, Sept. 2019.

[31] S. Ji, W.-Q. Wang, H. Chen, and Z. Zheng, "Secrecy capacity analysis of AN-aided FDA communication over Nakagami- $m$ fading," IEEE Wirel. Commun. Lett., vol. 7, no. 6, pp. 1034-1037, Dec. 2018.

[32] S. Ji and W.-Q. Wang, "Physical-layer security for frequency diverse array communication system over Nakagami- $m$ fading channels," IEEE Systems Journal, vol. 14, no. 2, pp. 2370-2381, Jun. 2020.

[33] J. M. Romero-Jerez, F. J. Lopez-Martinez, J. F. Paris, and A. J. Goldsmith, "The fluctuating two-ray fading model for mmWave communications," in Proceedings of 2016 IEEE Globecom Workshops, Washington, DC, USA, Dec. 2016, pp. 1-6.

[34] J. M. Romero-Jerez, F. J. Lopez-Martinez, J. F. Paris, and A. J. Goldsmith, "The fluctuating two-ray fading model: Statistical characterization and performance analysis," IEEE Trans. Wireless Commun., vol. 16, no. 7, pp. 4420-4432, Jul. 2017.

[35] J. Zhang, W. Zeng, X. Li, Q. Sun, and K. P. Peppas, "New results on the fluctuating two-ray model with arbitrary fading parameters and its applications," IEEE Trans. Veh. Technol., vol. 67, no. 3, pp. 2766-2770, Mar. 2018.

[36] O. S. Badarneh and D. B. da Costa, "Cascaded fluctuating two-ray fading channel,” IEEE Commun. Lett., vol. 23, no. 9, pp. 1497-1500, Sept. 2019.

[37] J. Zheng, J. Zhang, G. Pan, J. Cheng, and B. Ai, "Sum of squared fluctuating two-ray random variables with wireless applications," IEEE Trans. Veh. Technol., vol. 35, no. 8, pp. 8173-8177, Aug. 2019.

[38] W. Zeng, J. Zhang, S. Chen, K. P. Peppas, and B. Ai, "Physical layer security over fluctuating two-ray fading channels," IEEE Trans. Veh. Technol., vol. 67, no. 9, pp. 8949-8953, Sept. 2018.

[39] H. Zhao, L. Yang, G. Pan, and M.-S. Alouimi, "Secrecy outage analysis over fluctuating two-ray fading channels," Electro. Lett., vol. 55, no. 15 , pp. 866-868, Jul. 2019.

[40] M. Bilim and N. Kapucu, "Average symbol error rate analysis of QAM schemes over millimeter wave fluctuating two-ray fading channels," IEEE Access, vol. 7, pp. 105746-105754, Aug. 2019.

[41] H. Zhao, Z. Liu, and M.-S. Alouimi, "Different power adaption methods on fluctuating two-ray fading channels," IEEE Wirel. Commun. Lett., vol. 8, no. 2, pp. 592-595, Apr. 2019.

[42] J. Zheng, J. Zhang, S. Chen, H. Zhao, and B. Ai, "Wireless powered UAV relay communications over fluctuating two-ray fading channels," Physical Commun., vol. 35, pp. 1-8, May 2019.

$$
\begin{aligned}
\mathcal{B} & =\int_{0}^{\tau} \frac{\gamma_{\mathrm{E}}^{j_{\mathrm{E}}}}{\left(\tau-\gamma_{\mathrm{E}}\right)^{j_{\mathrm{E}}+2}} \frac{2^{n R_{0}}}{\left(2 \beta_{1}^{2} \sigma_{\mathrm{B}}^{2}\right)^{n}} \sum_{k=0}^{n}\left(\begin{array}{l}
n \\
k
\end{array}\right) \gamma_{\mathrm{E}}^{k}\left(\frac{2^{R_{0}}-1}{2^{R_{0}}}\right)^{n-k} \exp \left(-\frac{\gamma_{\mathrm{E}}}{2 \mu \sigma_{\mathrm{E}}^{2}\left(\tau-\gamma_{\mathrm{E}}\right)}-\frac{2^{R_{0}}\left(1+\gamma_{\mathrm{E}}\right)-1}{2 \beta_{1}^{2} \sigma_{\mathrm{B}}^{2}}\right) d \gamma_{\mathrm{E}} \\
& =\exp \left(-\frac{2^{R_{0}}-1}{2 \beta_{1}^{2} \sigma_{\mathrm{B}}^{2}}\right) \sum_{k=0}^{n}\left(\begin{array}{l}
n \\
k
\end{array}\right) \frac{2^{k R_{0}}\left(2^{R_{0}}-1\right)^{n-k}}{\left(2 \beta_{1}^{2} \sigma_{\mathrm{B}}^{2}\right)^{n}} \int_{0}^{\tau} \frac{\gamma_{\mathrm{E}}^{j_{\mathrm{E}}+k}}{\left(\tau-\gamma_{\mathrm{E}}\right)^{j_{\mathrm{E}}+2}} \exp \left(-\frac{\gamma_{\mathrm{E}}}{2 \mu \sigma_{\mathrm{E}}^{2}\left(\tau-\gamma_{\mathrm{E}}\right)}-\frac{2^{R_{0}} \gamma_{\mathrm{E}}}{2 \beta_{1}^{2} \sigma_{\mathrm{B}}^{2}}\right) d \gamma_{\mathrm{E}} \\
& =\exp \left(-\frac{2^{R_{0}}-1}{2 \beta_{1}^{2} \sigma_{\mathrm{B}}^{2}}\right) \sum_{k=0}^{n}\left(\begin{array}{l}
n \\
k
\end{array}\right) \frac{2^{k R_{0}}\left(2^{R_{0}}-1\right)^{n-k}}{\left(2 \beta_{1}^{2} \sigma_{\mathrm{B}}^{2}\right)^{n}} \Psi\left(0, j_{\mathrm{E}}+k, j_{\mathrm{E}}+2, \frac{2^{R_{0}}}{2 \beta_{1}^{2} \sigma_{\mathrm{B}}^{2}}, \frac{1}{2 \mu \sigma_{\mathrm{E}}^{2}}, \tau\right) .
\end{aligned}
$$


[43] P. Ioannides and C. A. Balanis, "Uniform circular and rectangular arrays for adaptive beamforming applications," IEEE Trans. Ante. \& Prop., vol. 4, pp. 351-354, Jul. 2005.

[44] C. Cetintepe and S. Demir, "Multipath characteristics of frequency diverse arrays over a ground plane," IEEE Trans. Ante. \& Prop., vol. 62, no. 7, pp. 3567-3574, Jul. 2014.

[45] H. Shao, J. Dai, J. Xiong, H. Chen, and W.-Q. Wang, "Dot-shaped rangeangle beampattern synthesis for frequency diverse array," IEEE Ante. \& Wirel. Propag. Lett., vol. 15, pp. 1703-1706, Feb. 2016.

[46] H. Yomo and E. D. Carvalho, "A CSI estimation method for wireless relay network," IEEE Commun. Lett., vol. 11, no. 6, pp. 480-482, Jun. 2007.

[47] H. Wu, Y. Jia, and S. B. Simmons, "Method and apparatus for LTE channel state information estimation," U.S. Patent US9294310B2, Mar. 222016.

[48] J. Xu, G. Liao, S. Zhu, L. Huang, and H. C. So, "Joint range and angle estimation using MIMO radar with frequency diverse array," IEEE Trans. Sig. Processing, vol. 63, no. 13, pp. 3396-3410, 2015.

[49] F. Shu, Y. Qin, T. Liu, L. Gui, Y. Zhang, and J. Li, "Low-complexity and high-resolution DOA estimation for hybrid analog and digital massive MIMO receive array," IEEE Trans. Commun., vol. 66, no. 6, pp. $2487-$ 2501, Jun. 2018.

[50] Y. Ding and V. Fusco, "Establishing metrics for assessing the performance of directional modulation systems," IEEE Trans. Ante. \& Propag., vol. 62, no. 5, pp. 2745-2755, May 2014.

[51] I. S. Gradshteyn and I. M. Ryzhik, Table of Integrals, Series, and Products, 7th ed. Academic Press, California, 2007.

[52] M. Abramowitz and I. A. Stegun, Handbook of Mathematical Functions with Formulas, Graphs, and Mathematical Tables, 1st ed. United States Department of Commerce, Washington D.C. New York, 1972.

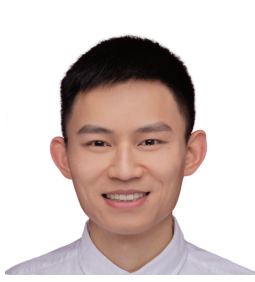

Qian Cheng received the B.S. degree in Communication Engineering from Xidian University, Xi'an, China, in July 2014, the M.S. degree in Information and Communication Engineering from the National University of Defense Technology (NUDT), Changsha, China, in December 2016, and the Ph.D. degree in Information and Communication Engineering from NUDT in December 2020, respectively.

$\mathrm{He}$ was a Visiting Ph.D. Researcher with the Institute of Electronics, Communications and Information Technology (ECIT), Queen's University Belfast from 2018 to 2020. His current research interests include physical layer security and directional modulation.

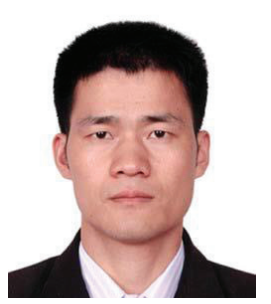

Shilian Wang received his B.S. and Ph.D. degrees in information and communication engineering from National University of Defense Technology (NUDT), Changsha, China, in 1998 and 2004, respectively. Since 2004, he continued research in wireless communications at NUDT, where he later became a Professor. From 2008 to 2009, he was a visiting scholar with the Department of Electronic and Electrical Engineering at Columbia University (CU), New York, USA

He is currently the Head of the Laboratory of Advanced Communication Technology at the College of Electronic Science and Technology, NUDT. His research interests include wireless communications and signal processing theory, physical layer security, spatial modulation, etc.

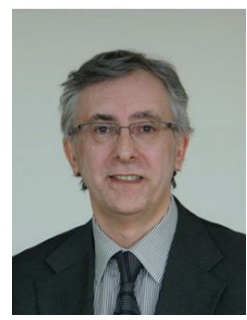

Vincent Fusco (S'82-M'82-SM'96-F'04) received the bachelor's degree (Hons.) in electrical and electronic engineering, the Ph.D. degree in microwave electronics, and the D.Sc. degree from Queen's University Belfast (QUB), Belfast, U.K., in 1979, 1982, and 2000 , respectively.

$\mathrm{He}$ is currently a Chief Technology Officer with the Institute of Electronics, Communications and Information Technology (ECIT), QUB. He has authored more than 450 scientific papers in major journals and in referred international conferences and two textbooks. He holds patents related to self-tracking antennas and has contributed invited papers and book chapters. His current research interests include advanced front-end architectures with enhanced functionality, active antenna, and front-end MMIC techniques.

Dr. Fusco is a Fellow of the Institution of Engineering and Technology, the Royal Academy of Engineers, and the Royal Irish Academy. He was a recipient of the IET Senior Achievement Award and the Mountbatten Medal, in 2012. He serves on the Technical Program Committee of various international conferences, including the European Microwave Conference.

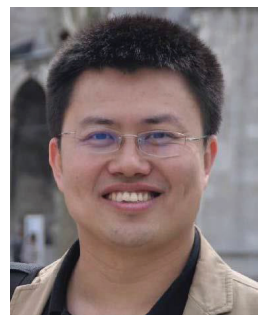

Fanggang Wang (S'10-M'11-SM'16) received the B.Eng. and Ph.D. degrees from the School of Information and Communication Engineering, Beijing University of Posts and Telecommunications, China, in 2005 and 2010, respectively. He was a PostDoctoral Fellow with the Institute of Network Coding, The Chinese University of Hong Kong, Hong Kong, from 2010 to 2012. He was a Visiting Scholar with the Massachusetts Institute of Technology from 2015 to 2016, and with the Singapore University of Technology and Design in 2014.

$\mathrm{He}$ is currently a Professor with the State Key Laboratory of Rail Traffic Control and Safety, School of Electronic and Information Engineering, Beijing Jiaotong University. His research interests are in wireless communications, signal processing, and information theory. He served as an Editor for the IEEE Communications Letters and a technical program committee member for several conferences.

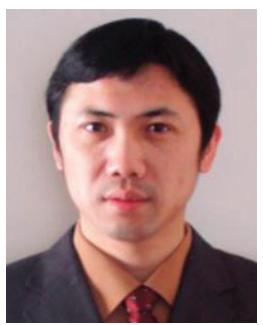

Jiang Zhu received the B.S., M.S., and Ph.D. degrees in electrical engineering from the National University of Defense Technology (NUDT), Changsha, China, in 1994, 1997, and 2000, respectively. From 2000 to 2004, he was a lecturer in communication engineering at the NUDT. He was a visiting scholar at the University of Calgary, AB, Canada from April 2004 to July 2005. From 2005 to 2008, he was an associate professor in communication engineering at the NUDT.

Since 2008, he has been with the NUDT as a full professor in the College of Electronic Science and Technology. His current research interests include wireless high speed communication technology, physical layer security and wireless sensor network.

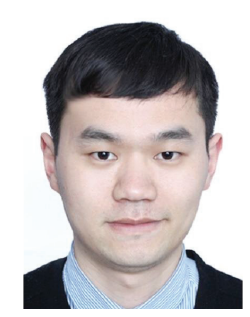

Chao Gu received the B.S. and M.S. degrees in electronic engineering from Xidian University, Xi'an, China, in 2009 and 2012, respectively, and the Ph.D. degree in electronic engineering at University of Kent, Canterbury, U.K., in 2017.

$\mathrm{He}$ is currently a senior engineer at the ECIT Institute, Queen's University Belfast, Belfast, U.K. His research interests include phased array antennas, reconfigurable antennas, and frequency selective surfaces. 